Sitientibus Série Ciências Físicas 05: 6 25 (2009)

\title{
O Campo de Radiação Quantizado sob Vínculos Macroscópicos e a Pressão de Casimir no Calibre de Coulomb
}

\section{The Quantized Radiation Field under Macroscopic Constrains and the Casimir Pressure in the Coulomb Gauge}

\author{
Franz A. Farias* \\ Departamento de Física - UEFS \\ Av. Transnordestina, s/n, Km 03, BR 116 \\ Feira de Santana - BA - 44036-900 \\ A. Matos Netd \\ Instituto de Fúsica - UFBA \\ Campus Universitário de Ondina \\ Salvador - BA - 40210-340
}

\begin{abstract}
O presente trabalho determina a pressão de Casimir seguindo as idéias da Eletrodinâmica Quântica Finita (Finite QED), i.e., construímos uma expressão para o tensor energiamomento (E-M) do campo de radiação quantizado de maneira a eliminar qualquer procedimento posterior de renormalização na obtenção de resultados finitos para os observáveis físicos, em particular, para a energia e o momento. O ponto de partida para este desenvolvimento é uma proposta de produto de operadores a ser utilizada na construção de observáveis, para os quais as medidas físicas sejam sempre resultados finitos. Com esta motivação, desenvolvemos o cálculo da pressão Casimir no calibre de Coulomb considerando dois tipos de condições de fronteira: a de Dirichlet e a de Neumann.
\end{abstract}

Palavras-chaves: Efeito Casimir, QED Finita, Calibre de Coulomb, CC Dirichlet, CC Neumann.

The present work determines the Casimir pressure by following the ideas of Finite Quantum Electrodynamics (Finite QED), that is, we propose an expression for the stress tensor of the quantized radiation field which intends eliminate any renormalization procedure to get finite results when carry on calculations of physical observables, in this case for energy and momentum. The starting point for this development is a proposal for the operator product to be used in the construction of the physical observables for which the physical measurements always are finite results. Taking this motivation we carry out the calculation of the Casimir pressure in the Coulomb gauge considering two types of boundary conditions: the Dirichlet one and the Neumann one.

Key-words: Casimir effect, Finite QED, Coulomb Gauge, Dirichlet BC, Neumann BC.

\section{INTRODUÇÃO}

Os operadores de campo locais, a exemplo de $A_{\mu}(x)$ são distribuições a valor-operador [1, 2, e uma vez que produtos de distribuições em um mesmo ponto, a exemplo de $A_{\mu}(x) A_{\nu}(x)$,

\footnotetext{
*Endereço Eletrônico: franz.farias@uefs.br

${ }^{\dagger}$ Endereço Eletrônico: arthurmn@ufba.br
}

não são bem definidos, estaremos sempre envolvidos com divergências associadas às singularidades que surgem desses cálculos. Estas últimas são usualmente referidas na literatura como as divergências ultravioletas.

No caso da energia, e mais geralmente as divergências (os valores esperados infinitos para as quantidades físicas) são tratadas, historicamente, de uma forma bastante pragmática. Uma vez que apenas diferenças de energia cons- 
tituem elementos mensuráveis o que se faz é redefinir a magnitude física a partir do nível de referência no infinito. Seguindo este argumento a expressão do operador físico $\hat{A}^{\mathrm{FIS}}$ é conseguida após uma apropriada substração de termos, procedimento usualmente designado por renormalização, que elimina o valor de vácuo infinito

$$
\left\langle\hat{A}^{\mathrm{FIS}}\right\rangle=\langle\hat{A}\rangle-\left\langle 0_{s v}|\hat{A}| 0_{s v}\right\rangle,
$$

onde $\left|0_{s v}\right\rangle$ é o estado quântico fundamental do sistema livre, i.e., do sistema quando não existem quaisquer vínculos (sistema livre).

Esta idéia de subtração foi estendida por H.B.G Casimir [3], em 1948, na determinação da parte finita da energia do estado fundamental do campo de radiação confinado, i.e., quando sob condições de fronteira. A renormalização de Casimir, de um ponto de vista matemático, é um "procedimento estranho", uma vez que envolve a subtração da grandeza física calculada em diferentes estados de vácuo, i.e, em estados pertencentes a diferentes espaços de Fock, e também porque estas grandezas têm intensidade infinita. Em outras palavras, no procedimento de renormalização de Casimir tem-se,

$$
\begin{aligned}
\left\langle 0_{c v}\left|\hat{A}^{\mathrm{FIS}}\right| 0_{c v}\right\rangle & \\
& =\left\langle 0_{c v}|\hat{A}| 0_{c v}\right\rangle-\left\langle 0_{s v}|\hat{A}| 0_{s v}\right\rangle,
\end{aligned}
$$

onde $\left|0_{c v}\right\rangle$ significa o estado de vácuo do sistema com vínculos, enquanto que $\left|0_{s v}\right\rangle$ significa o estado de vácuo do sistema sem vínculos.

Nossa proposta neste trabalho é estabelecer uma expressão apropriada para o tensor energia-momento (E-M) do campo eletromagnético de forma que consigamos resultados finitos para as grandezas físicas sem a necessidade de qualquer procedimento posterior de subtração (renormalização) e trabalhando todo o tempo no mesmo espaço de Fock. Assim, nosso ponto de partida é o procedimento cânonico usual baseado na definição do produto normal, i.e., do produto de Wick. Este programa foi realizado pela primeira vez para a densidade energia considerando o campo escalar sob o vínculo de Dirichlet [1, 4, 4].

Este trabalho [10] está estruturado em cinco seções. O efeito Casimir é discutido na seção 2, quando estabelecemos os vínculos sobre o campo eletromagnético em todos os casos de interesse. Na seção 3 introduzimos a proposta de produto de operador de campo e desenvolvemos a idéia do tensor de stress físico que tem sua forma independente da realização escolhida (espaço de Fock). A seção 4 contém os desenvolvimentos para a pressão de Casimir considerando vínculos sobre dois planos. Na seção 5 colocamos algumas observações e as conclusões, bem como uma comparação dos nossos resultados com aqueles conhecidos na literatura. No apêndice A estabelecemos os cálculos para a pressão de Casimir as condições de fronteira sobre um único plano, $x_{3}=0$. O apêndice $\mathrm{B}$ apresenta a reescrita das funções singulares $D_{c d}, D_{p d}$ e $D_{m t}$, que ocorrem nas situações de vínculos sobre dois planos, necessárias para os cálculos em termos das funções parciais de Jordan-Pauli $D_{0}^{+}\left(x_{0}, \mathbf{x}\right)$.

\section{SOBRE O EFEITO CASIMIR}

O interesse pelo efeito Casimir tem crescido desde a sua descoberta, e nas últimas décadas este modelo tem sido aplicado em contextos bastante distintos daquele originalmente descoberto (por exemplo: gravitação, nanoestruturas, geometria não-comutativa, entre outros).

De forma geral, as situações relacionadas em Teoria Quântica de Campos com condições de fronteira, campos externos ou modificações na geometria eou topologia do espaço-tempo tem sido usualmente designadas por efeito Casimir [11, 12]. Neste sentido, o modelo do efeito Casimir tem sido empregado, por exemplo, na elucidação do confinamento quark através do chamado modelo sacola (Bag model, MIT), como também na teoria quântica de campos em espaços-tempos curvos [13-15], ou seja, em presença de campos gravitacionais. Mais recentemente, o estudo do efeito Casimir tem também incluído a influência da temperatura $(T \neq 0)$ 
bem como correções devidas à rugosidade e à condutividade finita dos meios materiais [16].

Estamos interessados na pressão de Casimir do campo de radiação quantizado sob condições de fronteira macroscópicas. Para tanto, usaremos o tensor $T_{\mu \nu}(x)$ construído de maneira que seja desnecessário qualquer procedimento posterior de renormalização. A interação real, bastante complexa, entre o campo de radiação quantizado e as placas físicas reais que o confinam será modelada matematicamente por condições de fronteira sobre planos. Assim, uma placa uniforme e perfeitamente condutora será modelada, como usualmente [17, pela condição de fronteira de Dirichlet enquanto que uma placa uniforme e completamente permeável será modelada pela condição de fronteira de Neumann.

Consideramos os seguintes vínculos e as respectivas notações:

- O campo de radiação confinado por um plano perfeitamente condutor:

Vinculo Condutor Simples (CS)

$$
\left.\epsilon_{\mu \nu \rho \sigma} n^{\rho} F^{\sigma \nu}\right|_{x_{3}=0}=0
$$

- o campo de radiação confinado por um plano completamente permeável:

Vínculo Permeável Simples (PS)

$$
\left.\epsilon_{\mu \nu \rho \sigma} n^{\rho} \tilde{F}^{\sigma \nu}\right|_{x_{3}=0}=0,
$$

- o campo de radiação confinado por dois planos perfeitamente condutores: Vínculo Condutor Duplo (CD)

$$
\left\{\begin{array}{l}
\left.\epsilon_{\mu \nu \rho \sigma} n^{\rho} F^{\sigma \nu}\right|_{x_{3}=0}=0 \\
\left.\epsilon_{\mu \nu \rho \sigma} n^{\rho} F^{\sigma \nu}\right|_{x_{3}=d}=0
\end{array},\right.
$$

- o campo de radiação confinado por dois planos completamente permeáveis: Vínculo Permeável Duplo (PD)

$$
\left\{\begin{array}{c}
\left.\epsilon_{\mu \nu \rho \sigma} n^{\rho} \tilde{F}^{\sigma \nu}\right|_{x_{3}=0}=0 \\
\left.\epsilon_{\mu \nu \rho \sigma} n^{\rho} \tilde{F}^{\sigma \nu}\right|_{x_{3}=d}=0
\end{array},\right.
$$

- e o campo de radiação confinado por dois planos, um perfeitamente condutores e o outro completamente permeável:

Vínculo Misto (MT)

$$
\left\{\begin{array}{c}
\left.\epsilon_{\mu \nu \rho \sigma} n^{\rho} F^{\sigma \nu}\right|_{x_{3}=0}=0 \\
\left.\epsilon_{\mu \nu \rho \sigma} n^{\rho} \tilde{F}^{\sigma \nu}\right|_{x_{3}=d}=0
\end{array},\right.
$$

onde $\epsilon_{\mu \nu \rho \sigma}$ é o tensor de Levi-Civita completamente anti-simétrico e

$$
n^{\rho}=\left(\begin{array}{l}
0 \\
0 \\
0 \\
1
\end{array}\right)
$$

é um vetor normal unitário aos planos, $F^{\sigma \nu}$ é o tensor de campo eletromagnético e $\tilde{F}^{\sigma \nu}=$ $\frac{1}{2} \epsilon^{\sigma \nu \alpha \beta} F_{\alpha \beta}$ seu tensor dual. A dinâmica para o campo eletromagnético é descrita pela Lagrangiana

$$
\mathcal{L}=-\frac{1}{4} F_{\sigma \tau} F^{\sigma \tau}-\frac{\lambda}{2}\left(\partial_{\mu} A^{\mu}\right)^{2},
$$

com $\lambda$ sendo o termo fixador do calibre, de acordo com o procedimento usual de quantização em teoria de campo e

$$
\begin{aligned}
& {\left[A_{\mu}(\mathbf{r}, t), \partial_{t} A_{\nu}\left(\mathbf{r}^{\prime}, t\right)\right]} \\
& \quad=-i\left(g_{\mu \nu}+\frac{1}{\triangle} \partial_{\mu} \otimes \partial_{\nu}\right) \delta^{(3)}\left(\mathbf{r}-\mathbf{r}^{\prime}\right),
\end{aligned}
$$

com $\otimes$ indicando o produto tensorial entre os vetores. Lembramos que o tensor métrico $g_{\mu \nu}$ tem assinatura -2 . Escolheremos $\lambda=1$, i.e., o calibre de Feynman que dá como equação dinâmica

$$
\square A(x)=0 .
$$

Levamos em conta aqui o procedimento de Gupta-Bleuler para a métrica indefinida [18 que permite estabelecer o método canônico usual onde a expansão de $A_{\mu}(x)$, ou seja,

$$
A_{\mu}(x)=\sum_{i=1,2} \varepsilon_{\mu}^{(i)} \mathrm{f}_{(i)}(x) .
$$

Uma escolha apropriada para os vetores de polarização $\varepsilon_{\mu}^{(i)}$ torna-se necessária de forma a satisfazer cada uma das condições de fron- 
teira acima consideradas. As equações (3-7) mostram que as condições de fronteira atuam no espaço ortogonal aos vetores $n^{\rho}$ e $\partial_{\sigma}$ sobre $\mathrm{a}(\mathrm{s})$ superfície(s) $x_{3}=0\left(x_{3}=d\right)$. Uma vez que a covariância com respeito ao eixo $x_{3}$ é quebrada quando consideramos fronteiras (i.e., a invariância de Lorentz é quebrada quando incluimos os planos), a investigação da influência das fronteiras sobre o campo de radiação é simplificada com a escolha a seguir

$$
\begin{aligned}
& \varepsilon^{(0)}=\eta_{\rho}, \quad \varepsilon^{(1)}=\frac{1}{\sqrt{\triangle_{\perp} \triangle_{3}}}\left(\begin{array}{c}
0 \\
-\partial_{2} \partial_{3} \\
\partial_{1} \partial_{3} \\
0
\end{array}\right), \\
& \varepsilon^{(2)}=\frac{1}{\sqrt{\triangle_{\perp} \triangle}}\left(\begin{array}{c}
0 \\
\partial_{1} \partial_{3} \\
\partial_{2} \partial_{3} \\
-\triangle_{\perp}
\end{array}\right), \varepsilon^{(3)}=\frac{1}{\sqrt{\triangle}}\left(\begin{array}{c}
0 \\
\partial_{1} \\
\partial_{2} \\
\partial_{3}
\end{array}\right),
\end{aligned}
$$

onde

$$
\begin{aligned}
\triangle_{\perp} & \doteq \partial_{1}^{2}+\partial_{2}^{2}, \\
\triangle & \doteq \triangle_{\perp}+\triangle_{3} \doteq \partial_{1}^{2}+\partial_{2}^{2}+\partial_{3}^{2} .
\end{aligned}
$$

Os vetores de polarização satisfazem as seguintes propriedades

$$
\begin{aligned}
\sum_{p, q=1}^{3} \bar{g}_{p q} \varepsilon_{\mu}^{(p)} \otimes \varepsilon_{\nu}^{(q)} & =\bar{g}_{\mu \nu} \\
\sum_{p, q=1}^{2} \bar{g}_{p q} \varepsilon_{\mu}^{(p)} \otimes \varepsilon_{\nu}^{(q)} & =\bar{g}_{\mu \nu}+\frac{1}{\triangle} \bar{\partial}_{\mu} \otimes \bar{\partial}_{\nu},
\end{aligned}
$$

onde o tensor $\bar{g}_{\mu \nu}$ tem assinatura -3

$$
\bar{g}_{\mu \nu}=\left(\begin{array}{cccc}
0 & 0 & 0 & 0 \\
0 & -1 & 0 & 0 \\
0 & 0 & -1 & 0 \\
0 & 0 & 0 & -1
\end{array}\right)
$$

e $\bar{\partial}_{\mu} \doteq\left(0, \partial_{1}, \partial_{2}, \partial_{3}\right)$. Vale também que

$$
\varepsilon^{(p)} \otimes \varepsilon^{(q)}=\delta^{(p q)} .
$$

A simplificação mencionada com a escolha dos vetores de polarização em (12) refere-se ao estabelecimento de certas relaçôes que tornam-se válidas para todos os casos tratados aqui, mas isto nem sempre será pssível com outras escolhas, como veremos nas próximas seções.

\section{O TENSOR ENERGIA-MOMENTO FÍSICO}

O tensor de energia-momento (E-M) do campo eletromagnético é dado por [13, 18, 20]

$$
T_{\mu \nu}=\frac{1}{4} g_{\mu \nu} F_{\sigma \tau} F^{\sigma \tau}-F_{\mu}^{\rho} F_{\rho \nu},
$$

onde $F_{\mu \nu}=\partial_{\mu} A_{\nu}-\partial_{\nu} A_{\mu}$. Para trabalharmos de uma forma matematicamente consistente, empregaremos como um primeiro passo a técnica de separação de pontos [1, [3, 4, 11, 13, 14, 18, que permite definir $T_{\mu \nu}$ como

$$
\begin{aligned}
& T_{\mu \nu}(x)=\lim _{x^{\prime} \rightarrow x} {\left[\frac{1}{4} g_{\mu \nu} F_{\sigma \tau}(x) F^{\sigma \tau}\left(x^{\prime}\right)\right.} \\
&\left.-F^{\rho}{ }_{\mu}(x) F_{\rho \nu}\left(x^{\prime}\right)\right] .
\end{aligned}
$$

Desde que $A_{\sigma}(x)$ é uma função do ponto, cada termo do lado direito da Eq. 20 lê-se

$$
\begin{aligned}
& \frac{1}{4} g_{\mu \nu} F_{\sigma \tau}(x) F^{\sigma \tau}\left(x^{\prime}\right) \\
& =\Lambda_{\mu \nu}^{\rho \lambda \sigma \tau}\left(\partial_{\rho}^{x} \partial_{\lambda}^{x^{\prime}}\right)\left(A_{\sigma}(x) A_{\tau}\left(x^{\prime}\right)\right)
\end{aligned}
$$

com

$$
\begin{aligned}
\Lambda_{\mu \nu}^{\rho \lambda \sigma \tau} & =\frac{1}{2} g_{\mu \nu}\left(g^{\rho \lambda} g^{\sigma \tau}-g^{\rho \tau} g^{\sigma \lambda}\right),(22) \\
F_{\mu}^{\rho}(x) F_{\rho \nu}\left(x^{\prime}\right) & =\Omega_{\mu \nu}^{\rho \lambda \sigma \tau}\left(\partial_{\rho}^{x} \partial_{\lambda}^{x^{\prime}}\right)\left(A_{\sigma}(x) A_{\tau}\left(x^{\prime}\right)\right)
\end{aligned}
$$

e

$$
\begin{aligned}
\Omega_{\mu \nu}^{\rho \lambda \sigma \tau}= & -g_{\mu \alpha}\left(g^{\alpha \sigma} g^{\lambda \rho}-g^{\alpha \rho} g^{\lambda \sigma}\right) \delta_{\nu}^{\tau} \\
& -g_{\mu \alpha}\left(g^{\alpha \rho} g^{\tau \sigma}-g^{\alpha \sigma} g^{\tau \rho}\right) \delta_{\nu}^{\lambda}
\end{aligned}
$$

Substituindo (21) e (23) na Eq. 200 obtemos

$$
T_{\mu \nu}(x)=\lim _{x^{\prime} \rightarrow x}\left[G_{\mu \nu}^{\rho \lambda \sigma \tau}\right]\left(\partial_{\rho}^{x} \partial_{\lambda}^{x^{\prime}}\right)\left(A_{\sigma}(x) A_{\tau}\left(x^{\prime}\right)\right),
$$


onde $G_{\mu \nu}^{\rho \lambda \sigma \tau}$ tem a forma

$$
G_{\mu \nu}^{\rho \lambda \sigma \tau}=\Lambda_{\mu \nu}^{\rho \lambda \sigma \tau}+\Omega_{\mu \nu}^{\rho \lambda \sigma \tau}
$$

Um tensor energia-momento matematicamente consistente segue quando tomamos o produto de Wick (procedimento que é equivalente a retirada do infinito)

$$
\begin{aligned}
T_{\mu \nu}^{\mathrm{MAT}}(x)= & \lim _{x^{\prime} \rightarrow x}\left[G_{\mu \nu}^{\rho \lambda \sigma \tau}\right]\left(\partial_{\rho}^{x} \partial_{\lambda}^{x^{\prime}}\right) \times \\
& \times\left(: A_{\sigma}(x) A_{\tau}\left(x^{\prime}\right):\right) .
\end{aligned}
$$

Vamos considerar as situações em que os operadores de campo podem sempre ser covariantemente escritos como uma soma de duas partes, uma de freqüência positiva, a outra, negativa

$$
A_{\sigma}(x)=A_{\sigma}^{+}(x)+A_{\sigma}^{-}(x) .
$$

Isto permite-nos reescrever o produto normal

$$
\begin{aligned}
: A_{\sigma}(x) A_{\tau}\left(x^{\prime}\right): & =A_{\sigma}(x) A_{\tau}\left(x^{\prime}\right) \\
& -\left[A_{\sigma}^{+}(x), A_{\tau}^{-}\left(x^{\prime}\right)\right],
\end{aligned}
$$

portanto $T_{\mu \nu}^{\mathrm{MAT}}(x)$ lê-se

$$
\begin{aligned}
T_{\mu \nu}^{\mathrm{MAT}}(x) & =\lim _{x^{\prime} \rightarrow x}\left[G_{\mu \nu}^{\rho \lambda \sigma \tau}\right]\left(\partial_{\rho}^{x} \partial_{\lambda}^{x^{\prime}}\right) \times \\
& \times\left(A_{\sigma}(x) A_{\tau}\left(x^{\prime}\right)-\left[A_{\sigma}^{+}(x), A_{\tau}^{-}\left(x^{\prime}\right)\right]\right) .
\end{aligned}
$$

Partindo da expressão (30), nosso passo seguinte é estabelecer o tensor físico $T_{\mu \nu}^{\mathrm{FIS}}(x)$ que é independente da representação particular empregada, e isto é alcançado explicitando o resultado para o comutador em (29) na representação associada com o campo livre estendido a todo o espaço. Neste caso, $A_{\mu}(x)$ é

$$
\begin{gathered}
A_{\mu}(x)=\sum_{p=1}^{2} \varepsilon_{\mu}^{(p)} \int \frac{d^{3} k}{\sqrt{2 k_{0}(2 \pi)^{3}}} \times \\
\times\left[a_{(p)}(\mathbf{k}) \exp (-i k x)+a_{(p)}^{\dagger}(\mathbf{k}) \exp (i k x)\right],
\end{gathered}
$$

assumindo os vetores de polarização dados conforme a Eq. 12. Observando que

$$
\left[a_{(p)}(\mathbf{k}), a_{(q)}^{\dagger}\left(\mathbf{k}^{\prime}\right)\right]=-g_{p q} \delta^{(3)}\left(\mathbf{k}-\mathbf{k}^{\prime}\right)
$$

conseguimos para o segundo comutador no membro direito de 29),

$$
\begin{aligned}
& {\left[A_{\sigma}^{+}(x), A_{\tau}^{-}\left(x^{\prime}\right)\right]} \\
& =i \sum_{j, k=1}^{2} g_{j k} \varepsilon_{\sigma}^{(j)} \otimes \varepsilon_{\tau}^{\prime(k)} D_{0}^{+}\left(x-x^{\prime}\right),
\end{aligned}
$$

onde $D_{0}^{+}\left(x-x^{\prime}\right)$ é a parcela de frequência positiva da função de Jordan-Pauli $D_{0}\left(x-x^{\prime}\right)$ [1],

$D_{0}^{+}\left(x-x^{\prime}\right)=i \int \frac{d^{3} k}{(2 \pi)^{3} 2 k_{0}} \exp \left[-i k\left(x-x^{\prime}\right)\right]$.

Agora, é necessário conhecermos a atuação dos vetores $\varepsilon_{\tau}^{\prime(k)}$ (diferem $\operatorname{dos} \varepsilon_{\sigma}^{(j)}$ pois são derivadas em $x^{\prime}$ ) em (33) sobre a função $D_{0}^{+}\left(x-x^{\prime}\right)$, e como essa atuação pode ser reescrita em termos da atuação dos vetores $\varepsilon_{\tau}^{(k)}$ sobre a mesma função. Em outras palavras, desejamos substituir a ação dos vetores $\varepsilon_{\tau}^{\prime(k)}$ pelos $\varepsilon_{\tau}^{(k)}$. Para tanto, observamos que

$$
\begin{aligned}
\varepsilon_{\tau}^{\prime(1)} \exp \left(i k x^{\prime}\right) & \Leftrightarrow \varepsilon_{\tau}^{(1)} \exp (-i k x) \\
\varepsilon_{\tau}^{\prime(2)} \exp \left(i k x^{\prime}\right) & \Leftrightarrow \varepsilon_{\tau}^{(2)} \exp (-i k x) .
\end{aligned}
$$

Substituindo (33) e (35) na Eq. (30) obtemos a expressão final para o tensor $T_{\mu \nu}^{\mathrm{FIS}}$

$$
\begin{gathered}
T_{\mu \nu}^{\mathrm{FIS}}(x)=\lim _{x^{\prime} \rightarrow x}\left[G_{\mu \nu}^{\rho \lambda \sigma \tau}\right]\left(\partial_{\rho}^{x} \partial_{\lambda}^{x^{\prime}}\right)\left[A_{\sigma}(x) A_{\tau}\left(x^{\prime}\right)\right. \\
\left.-i\left(\bar{g}_{\sigma \tau}+\frac{1}{\triangle} \bar{\partial}_{\sigma} \otimes \bar{\partial}_{\tau}\right) D_{0}^{+}\left(x-x^{\prime}\right)\right]
\end{gathered}
$$

Este tensor tem a forma fisicamente requerida e exibe a invariância de Poincaré para o estado fundamental na ausência de fronteiras e/ou vínculos, ou seja,

$$
\left\langle T_{\mu \nu}^{\mathrm{FIS}}(x)\right\rangle_{s v} \equiv\left\langle 0_{s v}\left|T_{\mu \nu}^{\mathrm{FIS}}(x)\right| 0\right\rangle_{s v}=0,
$$

com $\left|0_{s v}\right\rangle$ sendo o estado fundamental do sistema campo estendido.

Na próxima seção determinamos a pressão de Casimir na presença de vínculos (Dirichlet e/ou Neumann) sobre duas fronteiras planas, paralelas e infinitas, aplicando a Eq. (36). 


\section{O EFEITO CASIMIR NA VARIEDADE DE BASE PLANA}

Iniciamos a modelagem da interação real entre o campo de radiação quantizado e as placas metálicas grossas (conforme apêndice A), que do ponto de vista quântico é, sem dúvida, nada trivial, através do modelo de planos sob a condição de Dirichlet. Esta modelagem considera, assim, o campo de radiação confinado por dois planos, perfeitamente condutores, paralelos, infinitos, um em $x_{3}=0$ e o outro em $x_{3}=d$, com $d$ sendo um número real positivo. Em particular, veremos que a condição de Dirichlet sobre os dois planos leva a uma força atrativa entre os planos que é macroscopicamente mensurável e que já foi objeto de medida experimental já em 1958 [21] e, mais tarde na década de 90 , medida com bastante precisão por Lamoreaux e Mohideen [22 24]; é o efeito Casimir original.

\section{A. O Vínculo Condutor Duplo (CD)}

A solução em todo o espaço para $A_{\mu}(x)$ é uma função por partes, definida em dada subregião, e que respeita o vínculo correspondente. As soluções para $A_{\mu}(x)$ nos semi-espaços infinitos foram estabelecidas no apêndice A. Por isto, focaremos a atenção na determinação do operador de campo e, por consequência, no valor esperado de vácuo de $T_{33}^{F I S}(x)$, para a região entre os planos.

As condições de fronteira em (5) são aquelas da seção 1 do Apêndice $\mathrm{A}$ em $x_{3}=0$ como também em $x_{3}=d$, logo as funções $\mathrm{fcd}_{(j)}(x)$ devem satisfazer

$$
\left.\partial_{3} \mathrm{f}_{(j)}^{\mathrm{cd}}(x)\right|_{\substack{x_{3}=0 \\ x_{3}=d}}=0 \quad(j=1,2) .
$$

A solução para o operador de campo, $A_{\mu}(x)$, em todo o espaço é

a) para $x_{3} \leq 0$,

A solução é dada pela Eq. A10;

b) para $0 \leq x_{3} \leq d$,

$A_{\mu}(x)=\sum_{p=1,2} \sum_{n=1}^{\infty} \varepsilon_{\mu}^{(p)} \int \frac{d^{2} k_{\perp}}{(2 \pi) \sqrt{2 k_{0}}} \sqrt{\frac{2}{d}} \cos \left[\frac{n \pi x_{3}}{d}\right]\left[\exp (-i \widetilde{k} \widetilde{x}) a_{(p)}\left(\mathbf{k}_{\perp}, n\right)+\exp (i \widetilde{k} \widetilde{x}) a_{(p)}^{\dagger}\left(\mathbf{k}_{\perp}, n\right)\right]$,

c) para $x_{3} \geq d$,

$$
A_{\mu}(x)=\sum_{p=1,2} \varepsilon_{\mu}^{(p)} \int \frac{d^{3} k}{(2 \pi)^{3 / 2} \sqrt{k_{0}}} \cos \left[k_{3}\left(x_{3}-d\right)\right]\left[\exp (-i \widetilde{k} \widetilde{x}) a_{(p)}(\mathbf{k})+\exp (i \widetilde{k} \widetilde{x}) a_{(p)}^{\dagger}(\mathbf{k})\right] .
$$

Na Eq. (39) o índice $n$ é um número inteiro. A fim de satisfazer a Eq. (9), os operadores de criação, $a$, e de aniquilação, $a^{\dagger}$, devem verificar as seguintes relações:

a) para as regiões $x_{3} \leq 0$ and $x_{3} \geq d$ vale as relações dadas em (A11), b) para a região $0 \leq x_{3} \leq d$,

$$
\begin{aligned}
{\left[a_{(p)}\left(\mathbf{k}_{\perp}, n\right), a_{(q)}^{\dagger}\left(\mathbf{k}_{\perp}^{\prime}, m\right)\right] } & =-g_{p q} \delta_{n m} \delta^{(2)}\left(\mathbf{k}_{\perp}-\mathbf{k}_{\perp}^{\prime}\right), \\
{\left[a_{p}\left(\mathbf{k}_{\perp}, n\right), a_{q}\left(\mathbf{k}_{\perp}^{\prime}, m\right)\right] } & =0, \\
{\left[a_{p}^{\dagger}\left(\mathbf{k}_{\perp}, n\right), a_{q}^{\dagger}\left(\mathbf{k}_{\perp}^{\prime}, m\right)\right] } & =0 .
\end{aligned}
$$


Podemos escrever o produto $A_{\sigma}(x) A_{\tau}\left(x^{\prime}\right)$ em função do produto normal relativo à representação associada de $A_{\sigma}(x)$ mais um comutador das parcelas de frequências positiva e negativa do referido operador em analogia ao que se faz no produto de Wick,

$$
\begin{aligned}
A_{\sigma}(x) A_{\tau}\left(x^{\prime}\right)= & \diamond A_{\sigma}(x) A_{\tau}\left(x^{\prime}\right) \diamond \\
& +\left[A_{\sigma}^{+}(x), A_{\tau}^{-}\left(x^{\prime}\right)\right],
\end{aligned}
$$

com a notação $\diamond A_{\sigma}(x) A_{\tau}\left(x^{\prime}\right) \diamond$ representando o ordenamento normal de operadores relativo ao estado de vácuo do espaço de Fock sob o vínculo em questão, i.e., o espaço de estados para o sistema confinado sob o vínculo (5). Portanto, calculando o comutador obtemos

$$
\begin{aligned}
& {\left[A_{\sigma}^{+}(x), A_{\tau}^{-}\left(x^{\prime}\right)\right]} \\
& \quad=i \sum_{j, l=1}^{2} \bar{g}_{j l} \varepsilon_{\sigma}^{(j)} \otimes \varepsilon_{\tau}^{\prime(l)} D_{c d}\left(\widetilde{x}-\widetilde{x}^{\prime} ; x_{3}, x_{3}^{\prime}\right) .
\end{aligned}
$$

A função $D_{c d}$ tem a seguinte forma

$$
\begin{aligned}
& D_{c d}\left(\widetilde{x}-\widetilde{x}^{\prime} ; x_{3}, x_{3}^{\prime}\right) \\
& =i \sum_{n=1}^{\infty} \int \frac{d^{2} k_{\perp}}{(2 \pi)^{2} k_{0} d} \cos \left[\frac{n \pi x_{3}}{d}\right] \cos \left[\frac{n \pi x_{3}^{\prime}}{d}\right] \times \\
& \quad \times \exp \left(-i \widetilde{k}\left(\widetilde{x}-\widetilde{x}^{\prime}\right)\right) .
\end{aligned}
$$

A próxima etapa no desenvolvimento é a escrita da função $D_{c d}$ em termos das funções $D_{0}^{+}$ (uma analogia ao método de imagens da eletrostática, com as múltiplas contribuições por reflexão). Conseguimos este objetivo usando a fórmula da soma de Poisson [10]

$$
\begin{aligned}
D_{c d} & \left(\tilde{x}-\tilde{x}^{\prime} ; x_{3}, x_{3}^{\prime}\right) \\
\quad= & \sum_{l=-\infty}^{\infty}\left[D_{0}^{+}\left(x-x_{2 l d}^{\prime}\right)+D_{0}^{+}\left(x-x_{2 l d}^{\prime \prime}\right)\right],
\end{aligned}
$$

que retoma a dependência exponencial nas variáveis $x_{3}$ e $x_{3}^{\prime}$; os detalhes encontram-se no Apêndice B. Os pontos $x_{2 l d}^{\prime}$ e $x_{2 l d}^{\prime \prime}$ significam

$$
\begin{aligned}
& x_{2 l d}^{\prime}=\left(x_{0}^{\prime}, x_{1}^{\prime}, x_{2}^{\prime}, x_{3}^{\prime}-2 l d\right), \\
& x_{2 l d}^{\prime \prime}=\left(x_{0}^{\prime}, x_{1}^{\prime}, x_{2}^{\prime},-x_{3}^{\prime}-2 l d\right) .
\end{aligned}
$$

Com a reescrita de $D_{c d}$ pela Eq. (46), e substituída em (44), levamos o resultado em (43) para a expressão do tensor energiamomento físico, Eq. (36), da qual extraímos o componente 33 como segue,

$$
\begin{aligned}
T_{33}^{\mathrm{FIS}}(x) & =\lim _{x^{\prime} \rightarrow x}\left[\sum_{i=1}^{3} \Gamma_{i}^{\sigma \tau}\right]\left[\diamond A_{\sigma}(x) A_{\tau}\left(x^{\prime}\right) \diamond\right. \\
& \left.+P_{\sigma \tau}^{(c d)}+Q_{\sigma \tau}^{(c d)}\right]
\end{aligned}
$$

$\operatorname{com} P_{\sigma \tau}^{(c d)}$ e $Q_{\sigma \tau}^{(c d)}$ dados por

$P_{\sigma \tau}^{(c d)}=i\left(\bar{g}_{\sigma \tau}+\frac{1}{\triangle} \bar{\partial}_{\sigma} \otimes \bar{\partial}_{\tau}\right) \sum_{\substack{-\infty \\(l \neq 0)}}^{\infty} D_{0}^{+}\left(x-x_{2 l d}^{\prime}\right)$,

$Q_{\sigma \tau}^{(c d)}=i \sum_{j, l=1}^{2} \bar{g}_{j l} \varepsilon_{\sigma}^{(j)} \otimes \varepsilon_{\tau}^{(l)} \sum_{l=-\infty}^{\infty} D_{0}^{+}\left(x-x_{2 l d}^{\prime \prime}\right)$.

O termo $l=0$, ausente em (50), foi cancelado pela contribuição oposta que compõe a definição do tensor energia-momento, $T_{\mu \nu}^{F I S}$, em (36), em particular seu componente $T_{33}^{F I S}$.

Observamos agora o desenvolvimento realizado no Apêndice A, após a Eq. A19 até a Eq. A22, que fornece neste caso,

$$
\begin{aligned}
i\left[\sum_{i=1}^{3} \Gamma_{i}^{\sigma \tau}\right] & \left(\sum_{j, l=1}^{2} \bar{g}_{j l} \varepsilon_{\sigma}^{(j)} \otimes \varepsilon_{\tau}^{\prime(l)}\right) \\
= & i\left[\left(\tilde{\partial}^{\sigma x^{\prime}} \tilde{\partial}_{\sigma}^{x}\right)+\left(\partial_{3}^{x^{\prime}} \partial_{3}^{x}\right)\right],
\end{aligned}
$$

enquanto que vale aqui também a Eq. A23

$$
\left[\left(\tilde{\partial}^{\sigma x^{\prime}} \tilde{\partial}_{\sigma}^{x}\right)+\left(\partial_{3}^{x^{\prime}} \partial_{3}^{x}\right)\right] D_{0}^{+}\left(x-x_{2 l d}^{\prime \prime}\right)=0 .
$$

Logo, a contribuição em (51), em razão da Eq. (53), é integralmente nula. Resta, então, a contribuição (50) que pode ser trabalhada tendo em conta a relação

$$
\begin{aligned}
& {\left[\sum_{i=1}^{3} \Gamma_{i}^{\sigma \tau}\right]\left(\bar{g}_{\sigma \tau}+\frac{1}{\triangle} \bar{\partial}_{\sigma} \otimes \bar{\partial}_{\tau}\right)} \\
& =-\left(\partial_{3}^{x} \partial_{3}^{x^{\prime}}\right)-\frac{1}{\triangle}\left(\partial_{3}^{x} \partial_{3}^{x}\right)\left(\partial_{0}^{x} \partial_{0}^{x^{\prime}}\right) .
\end{aligned}
$$


Tendo em conta as equações (53) e (54), o valor esperado de vácuo, associado ao vínculo condutor duplo, do componente do tensor energia-momento em $49, T_{33}^{\mathrm{FIS}}(x)$, dá

$$
\begin{aligned}
\left\langle T_{33}^{\mathrm{FIS}}(x)\right\rangle_{c d} & =-\lim _{x^{\prime} \rightarrow x} \sum_{\substack{-\infty \\
(l \neq 0)}}^{\infty}\left[\frac{1}{\triangle}\left(\partial_{3}^{x} \partial_{3}^{x}\right)\left(\partial_{0}^{x} \partial_{0}^{x^{\prime}}\right)\right. \\
& \left.+\left(\partial_{3}^{x} \partial_{3}^{x^{\prime}}\right)\right] D_{0}^{+}\left(x-x_{2 l d}^{\prime}\right) \cdot \quad(55)
\end{aligned}
$$

O cálculo da expressão acima é facilitado se observamos o fato a seguir

$$
\begin{array}{r}
-\frac{1}{\triangle}\left(\partial_{3}^{x} \partial_{3}^{x}\right)\left(\partial_{0}^{x} \partial_{0}^{x^{\prime}}\right) D_{0}^{+}\left(x-x_{2 l d}^{\prime}\right) \\
=-\left(\partial_{3}^{x} \partial_{3}^{x^{\prime}}\right) D_{0}^{+}\left(x-x_{2 l d}^{\prime}\right)
\end{array}
$$

e sendo assim, a Eq. 55 torna-se

$$
\begin{aligned}
& \left\langle T_{33}^{\mathrm{FIS}}(x)\right\rangle_{c d} \\
& =-2 \lim _{x^{\prime} \rightarrow x} \sum_{\substack{-\infty \\
(l \neq 0)}}^{\infty}\left(\partial_{3}^{x} \partial_{3}^{x^{\prime}}\right) D_{0}^{+}\left(x-x_{2 l d}^{\prime}\right) .
\end{aligned}
$$

Para avançarmos, vamos considerar uma forma mais apropriada para a função $D_{0}^{+}\left(x-x_{2 l d}^{\prime}\right)$ que é a sua representação no espaço de coordenadas [1],

$$
D_{0}^{+}\left(x_{0}, \mathbf{x}\right)=-\frac{i}{4 \pi^{2}}\left[\left(x_{0}-i \eta\right)^{2}-\mathbf{x}^{2}\right]^{-1},
$$

onde $\mathbf{x}=\left(x_{1}, x_{2}, x_{3}\right)$ é o vetor posição. Realizando as derivações em (57)

$$
\begin{aligned}
& -\left(\partial_{3}^{x} \partial_{3}^{x^{\prime}}\right) D_{0}^{+}\left(x-x_{2 l d}^{\prime}\right) \\
& =\frac{1}{2 \pi^{2}}\left[\left(x_{0}-x_{0}^{\prime}-i 0\right)^{2}-\left(\mathbf{x}-\mathbf{x}_{2 l d}^{\prime}\right)^{2}\right]^{-2} \\
& +\frac{2}{\pi^{2}} \frac{\left(x_{3}-x_{3}^{\prime}+2 l d\right)^{2}}{\left[\left(x_{0}-x_{0}^{\prime}-i 0\right)^{2}-\left(\mathbf{x}-\mathbf{x}_{2 l d}^{\prime}\right)^{2}\right]^{3}}
\end{aligned}
$$

e, em seguida, readequando o somatório e efetuando o limite $x^{\prime} \longrightarrow x$ conseguimos

$$
\left\langle T_{33}^{\mathrm{FIS}}(x)\right\rangle_{c d}=-\frac{3}{8 \pi^{2}}\left(\frac{1}{d^{4}}\right) \sum_{l=1}^{\infty} l^{-4} .
$$

A série infinita em (60) é a função zeta de Rie- mann $\zeta(z=3)$, cujo valor é $\pi^{4} / 90$ conforme [25]. O resultado final para a Eq. 60 é então

$$
\left\langle T_{33}^{\mathrm{FIS}}(x)\right\rangle_{c d}=-\frac{\pi^{2}}{240} \frac{\hbar c}{d^{4}} .
$$

Analisamos agora a pressão de Casimir, que é a pressão resultante, sobre cada um dos planos, em $x_{3}=0$ e $x_{3}=d$, de acordo as definições estabelecidas em (A1) e A29), mas com o ponto de vista da região interior, ou seja, para o plano $x_{3}=0$,

$$
\begin{aligned}
p(\tilde{x}, 0) & =\lim _{\delta \rightarrow 0}\left[\left\langle T_{33}^{\mathrm{FIS}}\left(\widetilde{x}, x_{3}=0+\delta\right)\right\rangle\right. \\
& \left.-\left\langle T_{33}^{\mathrm{FIS}}\left(\widetilde{x}, x_{3}=0-\delta\right)\right\rangle\right] \\
& =-\frac{\pi^{2}}{240} \frac{\hbar c}{d^{4}},
\end{aligned}
$$

enquanto que, para o plano em $x_{3}=d$ temos,

$$
\begin{aligned}
p(\tilde{x}, d) & =\lim _{\delta \rightarrow 0}\left[\left\langle T_{33}^{\mathrm{FIS}}\left(\widetilde{x}, x_{3}=d-\delta\right)\right\rangle\right. \\
& \left.-\left\langle T_{33}^{\mathrm{FIS}}\left(\widetilde{x}, x_{3}=d+\delta\right)\right\rangle\right] \\
& =-\frac{\pi^{2}}{240} \frac{\hbar c}{d^{4}} .
\end{aligned}
$$

Obtemos um fluxo de densidade de momento negativo, ou seja, a pressão é menor na região interior aos planos que na exterior, então existe uma força atrativa ente os mesmos. Este resultado está em concordância com aqueles conhecidos na literatura [3, 4, 11, 13, 14, 18].

\section{B. O Vínculo Permeável Duplo (PD)}

A condição de Neumann sobre dois planos infinitamente permeáveis (magneticamente) modelam duas placas grossas correspondentes. Nosso objetivo é determinar a pressão de Casimir neste confinamento com os planos separados por um distância $d$, em analogia à seção anterior. As condições de fronteira em (6) conduzem aos resultados em A30 e A31, valendo também para $x_{3}=d$,

$$
\left.\mathrm{f}_{(j)}^{\mathrm{pd}}(x)\right|_{\substack{x_{3}=0 \\ x_{3}=d}}=0, \quad \text { para } \quad(j=1,2),
$$


onde o índice $p d$ significa permeável duplo.

A expressão para o operador de campo
$A_{\mu}(x)$, em todo o espaço, para as condições de fronteira em (64) é

a) para $x_{3} \leq 0$,

A solução é dada pela Eq. A32;

b) para $0 \leq x_{3} \leq d$,

$A_{\mu}(x)=\sum_{p=1,2} \sum_{n=1}^{\infty} \varepsilon_{\mu}^{(p)} \int \frac{d^{2} k_{\perp}}{(2 \pi) \sqrt{2 k_{0}}} \sqrt{\frac{2}{d}} \operatorname{sen}\left[\frac{n \pi x_{3}}{d}\right]\left[\exp (-i \widetilde{k} \widetilde{x}) a_{(p)}\left(\mathbf{k}_{\perp}, n\right)+\exp (i \widetilde{k} \widetilde{x}) a_{(p)}^{\dagger}\left(\mathbf{k}_{\perp}, n\right)\right]$,

c) e para $x_{3} \geq d$

$A_{\mu}(x)=\sum_{p=1,2} \varepsilon_{\mu}^{(p)} \int \frac{d^{3} k}{(2 \pi)^{3 / 2} \sqrt{k_{0}}} \operatorname{sen}\left[k_{3}\left(x_{3}-d\right)\right]\left[\exp (-i \widetilde{k} \widetilde{x}) a_{(p)}(\mathbf{k})+\exp (i \widetilde{k} \widetilde{x}) a_{(p)}^{\dagger}(\mathbf{k})\right]$

observando as relações de comutações em (A11), 41) e 42.

Focamos a atenção na região entre os planos, pois as contribuições à pressão de Casimir advindas das regiões $x_{3} \leq 0$ e $x_{3} \geq d$ estão determinadas no Apêndice B. O procedimento aqui é análogo ao da seção anterior e o primeiro passo é a escrita do produto normal de operadores $\ddagger \ddagger$, tendo em conta o espaço de Fock relativo ao vínculo em questão. Com isto,

$$
\begin{aligned}
A_{\sigma}(x) A_{\tau}\left(x^{\prime}\right)= & \ddagger A_{\sigma}(x) A_{\tau}\left(x^{\prime}\right) \ddagger \\
& +\left[A_{\sigma}^{+}(x), A_{\tau}^{-}\left(x^{\prime}\right)\right] .
\end{aligned}
$$

O comutador em (67) é

$$
\begin{aligned}
& {\left[A_{\sigma}^{+}(x), A_{\tau}^{-}\left(x^{\prime}\right)\right]} \\
& \quad=i \sum_{j, l=1}^{2} \bar{g}_{j l} \varepsilon_{\sigma}^{(j)} \otimes \varepsilon_{\tau}^{\prime(l)} D_{p d}\left(\widetilde{x}-\widetilde{x}^{\prime} ; x_{3}, x_{3}^{\prime}\right),
\end{aligned}
$$

com a função $D_{p d}$ em 68 sendo

$$
\begin{aligned}
& D_{p d}\left(\widetilde{x}-\widetilde{x}^{\prime} ; x_{3}, x_{3}^{\prime}\right) \\
& =i \sum_{n=0}^{\infty} \int \frac{d^{2} k_{\perp}}{(2 \pi)^{2} k_{0} d} \operatorname{sen}\left[\frac{n \pi x_{3}}{d}\right] \operatorname{sen}\left[\frac{n \pi x_{3}^{\prime}}{d}\right] \times \\
& \quad \times \exp \left(-i \widetilde{k}\left(\widetilde{x}-\widetilde{x}^{\prime}\right)\right) .
\end{aligned}
$$

Precisamos agora reescrever 69 em termos das funções parciais de Jordan-Pauli $D_{0}^{+}(x-$ $\left.x^{\prime}\right)$. E, como já vimos, a reescrita desejada resulta da aplicação da fórmula de Poisson na Eq. (69), e este desenvolvimento encontra-se no Apêndice B. Logo,

$$
\begin{aligned}
D_{p d} & \left(\tilde{x}-\tilde{x}^{\prime} ; x_{3}, x_{3}^{\prime}\right) \\
\quad= & \sum_{l=-\infty}^{\infty}\left[D_{0}^{+}\left(x-x_{2 l d}^{\prime}\right)-D_{0}^{+}\left(x-x_{2 l d}^{\prime \prime}\right)\right],
\end{aligned}
$$

onde os pontos $x_{2 l d}^{\prime}$ e $x_{2 l d}^{\prime \prime}$ são aqueles em 477) e (48). Substituindo (68) em (67) e, em seguida, levando esta para a Eq. (36), obtemos para o componente 33 do tensor energia-momento,

$$
\begin{aligned}
T_{33}^{\mathrm{FIS}}(x) & =\lim _{x^{\prime} \rightarrow x}\left[\sum_{i=1}^{3} \Gamma_{i}^{\sigma \tau}\right]\left[\ddagger A_{\sigma}(x) A_{\tau}\left(x^{\prime}\right) \ddagger\right. \\
& \left.+P_{\sigma \tau}^{(p d)}+Q_{\sigma \tau}^{(p d)}\right]
\end{aligned}
$$

onde $P_{\sigma \tau}^{(p d)}$ e $Q_{\sigma \tau}^{(p d)}$ são

$P_{\sigma \tau}^{(p d)}=i\left(\bar{g}_{\sigma \tau}+\frac{1}{\triangle} \bar{\partial}_{\sigma} \otimes \bar{\partial}_{\tau}\right) \sum_{\substack{-\infty \\(l \neq 0)}}^{\infty} D_{0}^{+}\left(x-x_{2 l d}^{\prime}\right)$ 


$$
Q_{\sigma \tau}^{(p d)}=-i \sum_{j, l=1}^{2} \bar{g}_{j l} \varepsilon_{\sigma}^{(j)} \otimes \varepsilon_{\tau}^{\prime(l)} \sum_{l=-\infty}^{\infty} D_{0}^{+}\left(x-x_{2 l d}^{\prime \prime}\right)
$$

Notamos que fora o sinal negativo da contribuição (73) que é o que a difere da expressão análoga em (51), por sua vez a contribuição em (72) é exatamente a mesma que em (50). Disso decorre que os resultados a partir daqui são precisamente os mesmos que os da seção anterior, ou seja, temos uma contribuição nula de (73), que é resultante da validação da relação de dispersão de energia $k_{0}^{2}-\mathbf{k}^{2}=0$ sobre a função $D_{0}^{+}\left(x-x_{2 l d}^{\prime \prime}\right)$, enquanto que para a contribuição (72) temos (57) e, consequentemente, o rsultado final em (61).

Portanto, quando passamos ao vínculo permeável duplo, o que modela o confinamento do campo de radiação quantizado por duas placas magneticamente permeáveis, notamos que não há alteração na magnitude nem no sinal da pressão de Casimir, ou seja, a força de Casimir mantém seu caráter atrativo.

Assim, para o presente vínculo as equações 61), 62 e 63) são mantidas. Veremos a seguir, que no caso do vínculo misto, a força de Casimir muda seu caráter e torna-se repulsiva.

\section{O Vínculo Misto (MT)}

A situação que pretendemos modelar agora é o confinamento do campo de radiação quantizado por duas placas grossas (no sentido da discussão colocada na seção 1 do Apêndice A), uma placa condutora e outra magneticamente permeável. A modelagem matemática desse sistema é realizada com as condições de contorno mistas, i.e., pela condição de Dirichlet sobre o plano perfeitamente condutor em $x_{3}=0$, e pela condição de Neumann sobre o plano infinitamente permeável em $x_{3}=d$. Com isto, resulta a validação das equações em (A7) para o plano em $x_{3}=0$, enquanto que para o plano em $x_{3}=d$, temos a validação das equações em A30, ou seja,

$$
\left.\partial_{3} \mathrm{f}^{\mathrm{mt}}(j)(x)\right|_{x_{3}=0}=0, \quad \text { para } \quad(j=1,2) \text {, }
$$

e

$$
\left.\mathrm{f}^{\mathrm{mt}}{ }_{(j)}(x)\right|_{x_{3}=d}=0, \quad \text { para } \quad(j=1,2) .
$$

As funções $\mathrm{f}^{\mathrm{mt}}\left({ }_{j)}\right.$ expandem, portanto, $A_{\mu}(x)$ na região entre os planos. A realização de $A_{\mu}(x)$, por [10], nesta região $0 \leq x_{3} \leq d$ é

$$
A_{\mu}(x)=\sum_{p=1,2} \sum_{n=0}^{\infty} \varepsilon_{\mu}^{(p)} \int \frac{d^{2} k_{\perp}}{(2 \pi) \sqrt{k_{0} d}} \cos \left[\left(n+\frac{1}{2}\right) \frac{\pi x_{3}}{d}\right]\left[\exp (-i \widetilde{k} \widetilde{x}) a_{(p)}\left(\mathbf{k}_{\perp}, n\right)+\exp (i \widetilde{k} \widetilde{x}) a_{(p)}^{\dagger}\left(\mathbf{k}_{\perp}, n\right)\right]
$$

onde $n \geq 0$ é um número inteiro. Para a região $x_{3} \leq 0$,

$$
A_{\mu}(x) \text { é dado pela Eq. } A 10,
$$

enquanto que para $x_{3} \geq d$,

$$
A_{\mu}(x) \text { é dado pela Eq. 66. }
$$

Os operadores $a_{(p)}\left(\mathbf{k}_{\perp}, n\right)$ e $a_{(p)}^{\dagger}\left(\mathbf{k}_{\perp}, n\right)$ satisfazem as relações de comutação em (41) e 42.
As relações de comutação para os operadores correspondentes às regiões $x_{3} \leq 0$ e $x_{3} \geq d$ encontram-se determinadas na Eq. A11).

Do procedimento estabelecido na seção A e com a expressão para $A_{\mu}(x)$ em (76), reescrevemos o produto $A_{\sigma}(x) A_{\tau}\left(x^{\prime}\right)$ em função do produto normal de operadores relativo ao espaço

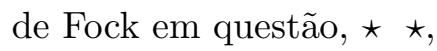

$$
\begin{aligned}
A_{\sigma}(x) A_{\tau}\left(x^{\prime}\right)= & \star A_{\sigma}(x) A_{\tau}\left(x^{\prime}\right) \star \\
& +\left[A_{\sigma}^{+}(x), A_{\tau}^{-}\left(x^{\prime}\right)\right],
\end{aligned}
$$


onde a notação $\star \star$ representa o produto normal relativo à nova representação. Calculando o comutador em (77) para a região $0 \leq x_{3} \leq d$, onde $A_{\mu}(x)$ é dado por (76), obtemos

$$
\begin{aligned}
& {\left[A_{\sigma}^{+}(x), A_{\tau}^{-}\left(x^{\prime}\right)\right]} \\
& \quad=i \sum_{j, l=1}^{2} \bar{g}_{j l} \varepsilon_{\sigma}^{(j)} \otimes \varepsilon_{\tau}^{\prime(l)} D_{m t}\left(\widetilde{x}-\widetilde{x}^{\prime} ; x_{3}, x_{3}^{\prime}\right),
\end{aligned}
$$

com a função $D_{m t}$ sendo dada por [10],

$$
\begin{aligned}
& D_{m t}\left(\tilde{x}-\tilde{x}^{\prime} ; x_{3}, x_{3}^{\prime}\right) \\
& \quad=i \sum_{n=0} \int \frac{d^{2} k_{\perp}}{(2 \pi)^{2} k_{0} d} \cos \left[\left(n+\frac{1}{2}\right) \frac{\pi x_{3}}{d}\right] \times \\
& \times \cos \left[\left(n+\frac{1}{2}\right) \frac{\pi x_{3}^{\prime}}{d}\right] \exp \left(-i \tilde{k}\left(\tilde{x}-\tilde{x}^{\prime}\right)\right) .
\end{aligned}
$$

Um desenvolvimento análogo aos da subseções A e B, como a reescrita de $D_{m t}$ em termos de $D_{0}^{+}\left(x-x^{\prime}\right)$ e da fórmula de Poisson (Apêndice B) resulta,

$$
\begin{aligned}
& D_{m t}\left(\tilde{x}-\tilde{x}^{\prime} ; x_{3}, x_{3}^{\prime}\right) \\
& =\sum_{l=-\infty}^{\infty}(-1)^{l}\left[D_{0}^{+}\left(x-x_{2 l d}^{\prime}\right)+D_{0}^{+}\left(x-x_{2 l d}^{\prime \prime}\right)\right] .
\end{aligned}
$$

Substituindo (80) em (78) e (77) em (36),

$$
\begin{aligned}
T_{33}^{\mathrm{FIS}}(x)= & \lim _{x^{\prime} \rightarrow x}\left[\sum_{i=1}^{3} \Gamma_{i}^{\sigma \tau}\right]\left[\star A_{\sigma}(x) A_{\tau}\left(x^{\prime}\right) \star\right. \\
& \left.+P_{\sigma \tau}^{(m t)}+Q_{\sigma \tau}^{(m t)}\right]
\end{aligned}
$$

com os termos $P_{\sigma \tau}^{(m t)}$ e $Q_{\sigma \tau}^{(m t)}$ sendo

$$
\begin{aligned}
P_{\sigma \tau}^{(m t)} & =i\left(\bar{g}_{\sigma \tau}+\frac{\bar{\partial}_{\sigma} \bar{\partial}_{\tau}}{\triangle}\right) \sum_{\substack{-\infty \\
(l \neq 0)}}^{\infty}(-1)^{l} D_{0}^{+}\left(x-x_{2 l d}^{\prime}\right), \\
Q_{\sigma \tau}^{(m t)} & =i \sum_{j, l=1}^{2} \bar{g}_{j l} \varepsilon_{\sigma}^{(j)} \otimes \varepsilon_{\tau}^{\prime(l)} \sum_{-\infty}^{\infty}(-1)^{l} D_{0}^{+}\left(x-x_{2 d}^{\prime \prime}\right) .
\end{aligned}
$$

O termo $l=0$ que não aparece em 82 foi simplificado pelo oposto correspondente na expressão do componente 33 do tensor energiamomento, $T_{33}^{\text {FIS }}(x)$, que segue de (36). Por outro lado, temos a manutenção das equações A23), que mostra uma contribuição nula advinda de (83), e (57), que simplifica o resultado do produto entre os $\Gamma_{i}^{\sigma \tau}$ e o termo $P_{\sigma \tau}^{(m t)}$. Dessa forma, tomando o valor esperado de vácuo, relativo ao vínculo misto, i.e., $\left|0_{m t}\right\rangle$ conseguimos,

$$
\begin{aligned}
& \left\langle T_{33}^{\mathrm{FIS}}(x)\right\rangle_{m t} \\
& =-2 \lim _{x^{\prime} \rightarrow x} \sum_{\substack{-\infty \\
(l \neq 0)}}^{\infty}\left(\partial_{3}^{x} \partial_{3}^{x^{\prime}}\right)(-1)^{l} D_{0}^{+}\left(x-x_{2 l d}^{\prime}\right) .
\end{aligned}
$$

O cálculo das derivadas em (84) segue de perto aquele realizado entre as equações (58) e 60), que neste caso dá

$$
\begin{aligned}
\left\langle T_{33}^{\mathrm{FS}}(x)\right\rangle_{m t} & \equiv\left\langle 0_{m t}\left|T_{33}^{\mathrm{FIS}}(x)\right| 0_{m t}\right\rangle \\
& =\frac{3}{8 \pi^{2} d^{4}} \sum_{l=1}^{\infty}(-1)^{l+1} \frac{1}{l^{4}} .
\end{aligned}
$$

A soma infinita acima é a função zeta de Riemann $\zeta(4)$ cujo valor é [25]

$$
\sum_{l=1}^{\infty}(-1)^{l+1} \frac{1}{l^{4}}=\left(\frac{7}{8}\right) \zeta(4)=\frac{7 \pi^{4}}{4 !}\left|B_{4}\right|,
$$

onde $B_{4}$ é o número de Bernoulli de índice 4 . Recuperando as unidades naturais e substituindo (86) em 85), obtemos

$$
\left\langle T_{33}^{\mathrm{FIS}}(x)\right\rangle_{m t}=\left(\frac{7}{8}\right) \frac{\pi^{2}}{240} \frac{\hbar c}{d^{4}}
$$

A pressão de Casimir em cada um dos planos, de acordo as definições estabelecidas em (A1) e A29, e com o ponto de vista da região interior, é para o plano $x_{3}=0$,

$$
\begin{aligned}
p(\tilde{x}, 0) & =\lim _{\delta \rightarrow 0}\left[\left\langle T_{33}^{\mathrm{FIS}}\left(\widetilde{x}, x_{3}=0+\delta\right)\right\rangle\right. \\
& \left.-\left\langle T_{33}^{\mathrm{FIS}}\left(\widetilde{x}, x_{3}=0-\delta\right)\right\rangle\right] \\
& =\left(\frac{7}{8}\right) \frac{\pi^{2}}{240} \frac{\hbar c}{d^{4}},
\end{aligned}
$$


enquanto que, para o plano em $x_{3}=d$ temos,

$$
\begin{aligned}
p(\tilde{x}, d) & =\lim _{\delta \rightarrow 0}\left[\left\langle T_{33}^{\mathrm{FIS}}\left(\widetilde{x}, x_{3}=d-\delta\right)\right\rangle\right. \\
& \left.-\left\langle T_{33}^{\mathrm{FIS}}\left(\widetilde{x}, x_{3}=d+\delta\right)\right\rangle\right] \\
& =\left(\frac{7}{8}\right) \frac{\pi^{2}}{240} \frac{\hbar c}{d^{4}} .
\end{aligned}
$$

$\mathrm{O}$ aspecto interessante e que distingue este dos casos até agora tratados é o sinal positivo que surge da contribuição para a pressão entre os planos, ou seja, a força tem agora um caráter repulsivo. Ademais, a magnitude da pressão corresponde a uma fração, precisamente $7 / 8$, do seu valor absoluto dado por (61). Mais uma vez, destacamos a concordância do resultado encontrado aqui com aqueles obtidos por outros autores utilizando diferentes métodos de cálculo [3, 4, 11, 13, 14, 18].

O caráter repulsivo da força de Casimir para a configuração de planos mistos remete ao resultado também conhecido na literatura para a variedade de base esférica (a geometria esférica), que exibe também uma força repulsiva [16, 33] e frustou uma idéia de H.B.G. Casimir em justificar a estabilidade do elétron, enquanto partícula carregada, a partir do efeito que leva hoje seu nome.

\section{ALGUMAS CONSIDERAÇÕES}

Este trabalho tem destacado que diferentes condições de fronteira implicam diferentes espaços de Fock associados ao campo e que, mais ainda, estes espaços, em particular, seus estados de vácuo quânticos respectivos, não são unitariamente equivalentes, portanto, a medida de um dado observável físico pode conduzir a resultados muito diferentes para diferentes condições de contorno [3, 26, 27]. Este reconhecimento pode ser melhor compreendido se observarmos que as condições de fronteira estabelecem um comportamento semelhante àquele devido aos campos externos, ou seja, no sentido que as condições de fronteira quebram simetrias e causam efeitos observáveis devido às flutuações quânticas [14].

Sendo assim, devemos observar no cálculo do observável físico a referência a um único espaço de estados (de Fock). Com isto apontamos para a necessidade de estabelecermos, como ponto de partida, uma expressão matematicamente consistente para o observável físico, que seja válida independentemente da condição de fronteira considerada (36) (ou ainda, dizemos das diferentes realizações para o campo eletromagnético). Assim, com a referência a um único espaço de estados, mostramos que o procedimento usual de renormalização (a subtração, ao final, dos valores esperados do observável físico tomados em diferentes estados de vácuo) torna-se desnecessário, o observável físico produz, ao final, um resultado finito.

A aplicação do tensor energia-momento físico em (36) revelou uma adequada regularização da expressão do observável físico, mas embora o método apresentado tenha sido bem sucedido na determinação da pressão de Casimir nas situações dos vínculos considerados, cabe atentarmos para alguns pontos. Primeiro, não realizamos o procedimento usual de renormalização no cálculo do observável, pois como mencionamos acima o resultado final foi finito em todos os casos, mas ocorre naturalmente uma normalização e que está construída de modo matematicamente consistente na expressão do observável físico, em particular para o tensor energia-momento em (36).

Segundo, a escolha dos vetores de polarização em (12) não foi arbitrária. Mesmo considerando a condição de transversalidade, existe ainda certa liberdade na escolha dos vetores e, infelizmente, algumas delas não permitem uma simplificação tal qual o procedimento estabelecido na seção 1 do Apêndice $A$ e na subseção $A$ da seção IV. Isto significa que a escolha dos vetores é governada pelo produto em (33) caso a caso de vínculo e, assim, nem sempre será possível garantir, para qualquer conjunto de vetores de polarização na forma (12), a simplificação ocorrida em (53).

Terceiro, como mencionamos antes, efetuamos uma modelagem simplificada para a interação bastante complexa entre o campo de radiação quantizado e as placas físicas, particularmente quando falamos das condu- 
toras, onde modelamos essas placas físicas por planos matemáticos sobre os quais é definida a condição de fronteira de Dirichlet. A modelagem que realizamos considera as placas como grossas [19, i.e., admitimos que os modos do campo de radiação que se propagam paralelamente às placas não penetram o condutor, caso contrário, com as placas sendo finas deveríamos considerar o fato de que aqueles modos atravessam a placa. Como procedemos, cada lado da placa foi tratado de maneira independente.

Nosso resultado para a condição de Dirichlet sobre os planos $x_{3}=0$ e $x_{3}=d$ reproduz aqueles obtidos por Brown e Maclay [32, Bordag, Robaschik e Wieczorek [19], e G. Scharf e W. F. Wreszinski [1. Os resultados para as situações permeável e mista estão em concordância com aqueles determinados por $\mathrm{T}$. Boyer pelo método de soma de modos [33. Estas comparações demonstram que o método utilizado aqui é confiável, mas não temos idéia a respeito da sua aplicabilidade em outras variedades de base, a exemplo do cilindro e da esfera. O fato que os vetores de polarização na representação de coordenadas nestas variedades de base dependam das coordenadas espaciais pode constituir em um elemento de maior complexidade na execução dos cálculos necessários.

\section{Agradecimentos}

Os autores agradecem as discussões valiosas e esclarecedoras do Prof. Dr. Ademir E. Santana (Instituto de Física da Universidade de Brasília (UnB)).

\section{APÊNDICE A: O EFEITO CASIMIR SOBRE UM ÚNICO PLANO}

O efeito Casimir sobre um único plano não será tratado como caso limite da situação de dois planos embora esta abordagem possa ser considerada (i.e. teríamos um processo limite tomado apropriadamente, ou seja, levaríamos um dos planos infinitamente distanciado do outro). Preferimos deixar explícitos os cálculos nesses casos, os de vínculo sobre um plano, em razão do estabelecimento das notações, definições e equações gerais que, como o nome mesmo se refere, valerão inclusive para as situações com dois planos, nas regiões dos semiespaços.

Vamos desenvolver os cálculos desde o início, pois as expressões para estes casos são necessárias para a determinação da pressão de Casimir quando são envolvidos dois planos, a exemplo da expressão do operador de campo, $A_{\mu}(x)$, em todo o espaço. Fazendo desta forma ficamos envolvidos, nas situações de vínculo sobre dois planos, com a determinação da expressão do operador de campo apenas para a região entre os planos.

O "confinamento" do campo eletromagnético por um único plano infinito, como é esperado, deve conduzir a um resultado nulo para a pressão de Casimir, em razão da simetria no espetro dos modos normais de vibração produzido pelo vínculo nas duas regiões em que o espaço fica divido, i.e., embora discretizado, o espectro de frequências possíveis do campo eletromagnético é exatamente o mesmo para cada região do semi-espaço infinito definido pela presença do plano em $x_{3}=0$, com isso a pressão resultante sobre o plano é nula.

A quantização do campo eletromagnético considerada aqui admite que as placas físicas são espessas [19], i.e., as placas são suficientemente grossas de modo a confinar o campo de radiação na região de interesse, portanto não consideramos a possibilidade de propagação do mesmo através da placa. Isto implica a contagem do modo $n=0$ nas funções que satisfazem a condição de fronteira $\left.\partial_{3} \mathrm{f}_{j}\right|_{S}=0$.

Vamos admitir, portanto, que o vínculo físico constituído por uma placa material é modelada por um plano no qual vale uma das condições de contorno, a de Dirichlet (3) ou a de Neumann (4), conforme desejemos modelar uma placa perfeitamente condutora ou uma infinitamente permeável, respectivamente. $\mathrm{Na}$ próxima subseção explicitamos o cálculo da pressão de Casimir para uma fronteira perfeitamente condutora. O cálculo para a fronteira infinitamente permeável é desenvolvido na subseção seguinte. 


\section{O Vínculo Condutor Simples (CS)}

O entendimento que adotamos para a pressão de Casimir é o de que esta pressão seja a pressão resultante [19]. Contudo, independende do valor encontrado para $\left\langle T_{33}^{\mathrm{FIS}}(x)\right\rangle_{c v}$ em dado semi-espaço, ele será o mesmo na outra região, pois o espaço foi dividido pelo plano, e a pressão resultante é, portanto, nula

$$
\begin{aligned}
p(\tilde{x}, 0)= & \lim _{\delta \rightarrow 0}\left[\left\langle T_{33}^{\mathrm{FIS}}\left(\widetilde{x}, x_{3}=0+\delta\right)\right\rangle\right. \\
& \left.-\left\langle T_{33}^{\mathrm{FIS}}\left(\widetilde{x}, x_{3}=0-\delta\right)\right\rangle\right]=0,
\end{aligned}
$$

$\operatorname{com} \tilde{x}=\left(x_{0}, x_{1}, x_{2}, 0\right)$. Da Eq. (36) temos

$$
\begin{gathered}
T_{33}^{\mathrm{FIS}}(x)=\lim _{x^{\prime} \rightarrow x}\left[G_{33}^{\rho \lambda \sigma \tau}\right]\left(\partial_{\rho}^{x} \partial_{\lambda}^{x^{\prime}}\right)\left(A_{\sigma}(x) A_{\tau}\left(x^{\prime}\right)\right. \\
\left.-i\left(\bar{g}_{\sigma \tau}+\frac{1}{\triangle} \bar{\partial}_{\sigma} \bar{\partial}_{\tau}\right) D_{0}^{+}\left(x-x^{\prime}\right)\right),
\end{gathered}
$$

onde $\left[G_{33}^{\rho \lambda \sigma \tau}\right]\left(\partial_{\rho}^{x} \partial_{\lambda}^{x^{\prime}}\right)$ em $\mathrm{A} 22$ é

$$
\left[G_{33}^{\rho \lambda \sigma \tau}\right]\left(\partial_{\rho}^{x} \partial_{\lambda}^{x^{\prime}}\right)=\sum_{i=1}^{3} \Gamma_{i}^{\sigma \tau}
$$

com os operadores $\Gamma_{1}^{\sigma \tau}, \Gamma_{2}^{\sigma \tau}$ e $\Gamma_{3}^{\sigma \tau}$ sendo

$$
\begin{aligned}
& \Gamma_{1}^{\sigma \tau}=\left(g^{3 \sigma} \delta_{3}^{\tau}-\frac{1}{2} g^{\sigma \tau}\right)\left(\partial^{\lambda x} \partial_{\lambda}^{x^{\prime}}\right),(\mathrm{A} 4) \\
& \Gamma_{2}^{\sigma \tau}=\frac{1}{2}\left(\partial^{\tau x} \partial^{\sigma x^{\prime}}\right)+\delta_{3}^{\tau}\left(\partial_{3}^{x} \partial^{\sigma x^{\prime}}\right)-g^{3 \sigma}\left(\partial^{\tau x} \partial_{3}^{x^{\prime}}\right), \\
& \Gamma_{3}^{\sigma \tau}=-g^{\sigma \tau}\left(\partial_{3}^{x} \partial_{3}^{x^{\prime}}\right) .
\end{aligned}
$$

A condição de fronteira em (3) para o vínculo condutor simples torna-se,

$$
\begin{aligned}
&\left.\frac{1}{\sqrt{\triangle_{\perp}}} \triangle_{\perp} \partial_{3} \mathrm{f}^{\mathrm{cs}}{ }_{(1)}(x)\right|_{x_{3}=0}=0 \\
& \frac{\partial_{0} \partial_{1} \partial_{3} \mathrm{fss}_{(1)}(x)}{\sqrt{\triangle_{\perp}}}+\left.\frac{\partial_{0} \partial_{2} \partial_{3} \mathrm{f}^{\mathrm{cs}}{ }_{(2)}(x)}{\sqrt{\triangle_{\perp} \triangle}}\right|_{x_{3}=0}=0 \\
&-\frac{\partial_{0} \partial_{2} \partial_{3} \mathrm{fs}^{\mathrm{cs}}(x)}{\sqrt{\triangle_{\perp}}}+\left.\frac{\partial_{0} \partial_{1} \partial_{3} \mathrm{f}^{\mathrm{cs}}{ }_{(2)}(x)}{\sqrt{\triangle_{\perp} \triangle}}\right|_{x_{3}=0}=0,
\end{aligned}
$$

e, uma vez que as funções $\mathrm{f}_{(i)}(x)$ são independentes uma das outras resulta,

$$
\left.\partial_{3} \mathrm{fcs}_{(j)}(x)\right|_{x_{3}=0}=0, \quad \text { para } \quad(j=0,1,2) .
$$

Agora aplicamos a Eq. A2 considerando a representação de $A_{\sigma}(x)$ relacionada ao vínculo de interesse [10. A solução de (10) sob a condição de fronteira (3) é conseguida utilizando o método de separação de variáveis, enquanto que os fatores de normalização das funções resultam da condição de completeza e ortonormalidade, que se expressa

$$
\begin{gathered}
\qquad \sum_{j=1}^{2} \int d^{3} \mathbf{r} \mathrm{f}_{(j)\left(\tilde{k}^{\prime}, k_{3}^{\prime}\right)}^{-}(x)\left[i \overleftrightarrow{\partial_{0}}\right] \mathrm{f}_{(j)\left(\tilde{k}, k_{3}\right)}^{+}(x) \\
=\delta^{(3)}\left(\mathbf{k}-\mathbf{k}^{\prime}\right) \\
\text { onde } A\left[\overleftrightarrow{\partial_{\nu}}\right] B=A\left(\partial_{\nu} B\right)-\left(\partial_{\nu} A\right) B .
\end{gathered}
$$

Consideramos um plano perfeitamente condutor situado em $x_{3}=0$ e, portanto, o espaço fica dividido em duas regiões (desconexas) $x_{3} \leq$ 0 e $x_{3} \geq 0$. Para distinguirmos as regiões introduzimos um índice nos operadores de criação e aniquilação, designado por $\chi$, e cujos valores são $E$ para a região $x_{3} \leq 0$ e $D$ para a região $x_{3} \geq 0$. O operador de campo $A_{\mu}(x)$ assume, então, a seguinte forma

$$
\begin{aligned}
& A_{\mu}(x)=\sum_{p} \varepsilon_{\mu}^{(p)} \int \frac{d^{3} k}{(2 \pi)^{3 / 2} \sqrt{k_{0}}} \cos \left(k_{3} x_{3}\right) \times \\
& \times\left[\exp (-i \widetilde{k} \widetilde{x}) a_{(p) \chi}(\mathbf{k})+\exp (i \widetilde{k} \widetilde{x}) a_{(p) \chi}^{\dagger}(\mathbf{k})\right]
\end{aligned}
$$

Os operadores $a_{(p) \chi}(\mathbf{k})$ e $a_{(p) \chi}^{\dagger}(\mathbf{k})$ satisfazem as seguintes relações de comutação

$\left[a_{(p) \chi}(\mathbf{k}), a_{(q) \chi^{\prime}}^{\dagger}\left(\mathbf{k}^{\prime}\right)\right]=-\bar{g}_{p q} \delta_{\chi \chi^{\prime}} \delta^{(3)}\left(\mathbf{k}-\mathbf{k}^{\prime}\right)$,
$\left[a_{(p) \chi}(\mathbf{k}), a_{(q) \chi^{\prime}}\left(\mathbf{k}^{\prime}\right)\right]=\left[a_{(p) \chi}^{\dagger}(\mathbf{k}), a_{(q) \chi^{\prime}}^{\dagger}\left(\mathbf{k}^{\prime}\right)\right]=0$.

O próximo passo é escrevermos o produto de operadores $A_{\sigma}(x) A_{\tau}\left(x^{\prime}\right)$ em função do produto normal relativo à representação de $A_{\sigma}(x)$ que está associada ao espaço de Fock gerado pelo vetor cíclico $\left|0_{c s}\right\rangle$, ou seja, seguindo o de- 
senvolvimento estabelecido na seção III, entre as equações 27, 30,

$$
\begin{aligned}
A_{\sigma}(x) A_{\tau}\left(x^{\prime}\right)= & \circ A_{\sigma}(x) A_{\tau}\left(x^{\prime}\right) \circ \\
& +\left[A_{\sigma}^{+}(x), A_{\tau}^{-}\left(x^{\prime}\right)\right],
\end{aligned}
$$

Chamamos a atenção para a notação "o o" que representa o produto normal entre operadores associados ao espaço de Fock definido pelo vínculo condutor simples. Calculando o comutador obtemos

$$
\begin{aligned}
& {\left[A_{\sigma}^{+}(x), A_{\tau}^{-}\left(x^{\prime}\right)\right]} \\
& \quad=i \sum_{j, l=1}^{2} \bar{g}_{j l} \varepsilon_{\sigma}^{(j)} \otimes \varepsilon_{\tau}^{\prime(l)} D_{2}^{+}\left(\widetilde{x}-\widetilde{x}^{\prime} ; x_{3}, x_{3}^{\prime}\right),
\end{aligned}
$$

com a função $D_{2}^{+}$significando

$$
\begin{aligned}
D_{2}^{+} & \left(\widetilde{x}-\widetilde{x}^{\prime} ; x_{3}, x_{3}^{\prime}\right) \\
& =i \int \frac{d^{3} k}{(2 \pi)^{3} k_{0}} \cos \left(k_{3} x_{3}\right) \cos \left(k_{3} x_{3}^{\prime}\right) \times \\
& \times \exp \left[-i \widetilde{k}\left(\widetilde{x}-\widetilde{x}^{\prime}\right)\right] .
\end{aligned}
$$

A função $D_{2}^{+}$pode, usando a fórmula de Poisson [10, ser reescrita

$D_{2}^{+}\left(\tilde{x}-\tilde{x}^{\prime} ; x_{3}, \tilde{x}_{3}\right)=D_{0}^{+}\left(x-x^{\prime}\right)+D_{0}^{+}\left(x-x^{\prime \prime}\right)$

onde os pontos $x^{\prime}$ e $x^{\prime \prime}$ são

$$
\begin{aligned}
x^{\prime} & =\left(x_{0}^{\prime}, x_{1}^{\prime}, x_{2}^{\prime}, x_{3}^{\prime}\right) \\
x^{\prime \prime} & =\left(x_{0}^{\prime}, x_{1}^{\prime}, x_{2}^{\prime},-x_{3}^{\prime}\right) .
\end{aligned}
$$

Levamos agora a expressão A15 para (A13) e, em seguida, substituímos (A12) em (A2), observando a identificação em (33). Ocorre também uma simplificação para a combinação dos vetores de polarização que atuam sobre $D_{0}^{+}\left(x-x^{\prime \prime}\right)$, assim,

$$
\begin{array}{r}
T_{33}^{\mathrm{FIS}}(x)=\lim _{x^{\prime} \rightarrow x}\left[\sum_{i=1}^{3} \Gamma_{i}^{\sigma \tau}\right]\left[\circ A_{\sigma}(x) A_{\tau}\left(x^{\prime}\right) \circ\right. \\
\left.+i \sum_{j, l=1}^{2} \bar{g}_{j l} \varepsilon_{\sigma}^{(j)} \otimes \varepsilon_{\tau}^{\prime(l)} D_{0}^{+}\left(x-x^{\prime \prime}\right)\right] .
\end{array}
$$

Quando tomamos o valor esperado da Eq. A18 no estado de vácuo correspondente, i.e., $\left|0_{c s}\right\rangle$, conseguimos mais uma simplificação, pois o primeiro termo dá contribuição nula. Portanto, resta só o segundo termo,

$$
\begin{aligned}
& \left\langle T_{33}^{\mathrm{FIS}}(x)\right\rangle_{c s}=-i \lim _{x^{\prime} \rightarrow x}\left[\sum_{i=1}^{3} \Gamma_{i}^{\sigma \tau}\right] \times \\
& \times\left(\varepsilon_{\sigma}^{(1)} \otimes \varepsilon_{\tau}^{\prime(1)}+\varepsilon_{\sigma}^{(2)} \otimes \varepsilon_{\tau}^{\prime(2)}\right) D_{0}^{+}\left(x-x^{\prime \prime}\right) .
\end{aligned}
$$

O cálculo da expressão acima depende do conhecimento da atuação dos vetores $\varepsilon_{\tau}^{\prime(j)}$ sobre a função $D_{0}^{+}\left(x-x^{\prime \prime}\right)$, numa maneira análoga ao que fizemos na seção III, pg. 5, quando observamos a atuação daqueles sobre a função $D_{0}^{+}\left(x-x^{\prime}\right)$. O estudo dessa atuação nos leva às seguintes identidades

$$
\begin{aligned}
\varepsilon_{\tau}^{\prime(1)} \exp \left(i k x^{\prime \prime}\right) \Leftrightarrow & -\varepsilon_{\tau}^{(1)} \exp (-i k x) \\
\varepsilon_{\tau}^{\prime(2)} \exp \left(i k x^{\prime \prime}\right) \Leftrightarrow & -\varepsilon_{\tau}^{(2)} \exp (-i k x) \\
& -\frac{2 \triangle_{\perp}}{\sqrt{\triangle_{\perp} \triangle}} \eta_{\tau} \exp (-i k x) .
\end{aligned}
$$

Com as identidades anteriores obtemos uma contribuição já encontrada na seção III mais uma nova contribuição advinda do último termo da segunda identidade em A20. Por sua vez, os operadores $\Gamma_{i}^{\sigma \tau}$ encontram-se definidos pelas equações (A4)- A6). Calculando os produtos obtemos:

$$
\begin{aligned}
& -\left[\sum_{i=1}^{3} \Gamma_{i}^{\sigma \tau}\right]\left(\bar{g}_{\sigma \tau}+\frac{1}{\triangle} \bar{\partial}_{\sigma} \bar{\partial}_{\tau}\right) \\
& =\left(\partial_{3}^{x} \partial_{3}^{x^{\prime}}\right)+\frac{1}{\triangle}\left(\partial_{3}^{x} \partial_{3}^{x}\right)\left(\partial_{0}^{x} \partial_{0}^{x^{\prime}}\right),
\end{aligned}
$$

enquanto que para a segunda parte

$$
\begin{gathered}
{\left[\sum_{i=1}^{3} \Gamma_{i}^{\sigma \tau}\right]\left(\frac{2 \triangle_{\perp}}{\sqrt{\triangle_{\perp} \triangle}} \eta_{\tau}\right)} \\
=\frac{\triangle_{\perp}}{\triangle}\left(\partial_{3}^{x} \partial_{3}^{x^{\prime}}\right)+\frac{\triangle_{\perp}}{\triangle}\left(\partial^{\lambda x} \partial_{\lambda}^{x^{\prime}}\right) \\
-\frac{1}{\triangle}\left(\partial_{3}^{x} \partial_{3}^{x}\right)\left(\partial_{1}^{x} \partial_{1}^{x^{\prime}}+\partial_{2}^{x} \partial_{2}^{x^{\prime}}\right) .
\end{gathered}
$$


Reunindo as contribuições A21 e A22 e efetuando as derivações sobre a função $D_{0}^{+}\left(x-x^{\prime \prime}\right)$ expressa no espaço dos momentos alcançamos como resultado

$$
\left[\left(\tilde{\partial}^{\lambda x} \tilde{\partial}_{\lambda}^{x^{\prime}}\right)+\left(\partial_{3}^{x} \partial_{3}^{x^{\prime}}\right)\right] D_{0}^{+}\left(x-x^{\prime \prime}\right)=0
$$

A Eq. (A19) simplifica-se, assim, trivialmente

$$
\left\langle T_{33}^{\mathrm{FIS}}(x)\right\rangle_{c s}=0
$$

e a pressão de Casimir tem o resultado esperado como dado pela Eq. A1).

Chamamos a atenção para o desenvolvimento entre as equações (A20) e (A23), porque o resultado em (A23) é geral e, portanto, aplicável toda vez que surgir a combinação entre os $\Gamma_{\sigma \tau}^{i}$ e os vetores de polarização $\varepsilon^{(j)}$ tal como encontrada em (A18). Este processo é, sem dúvida, um facilitador, pois simplifica o cálculo das contribuições em todos os casos considerados neste trabalho.

Por outro lado, como também consideraremos da região do semi-espaço infinito $x_{3} \geq d$ definida com o plano em $x_{3}=d$, devemos observar as modificações nas expressões gerais que ocorrem em função desse deslocamento espacial do plano. Destacamos, entretanto, que não esperamos encontrar qualquer alteração no resultado final obtido em A24, uma vez que o resultado físico, por simetria, deve ser invariante com respeito a este deslocamento. Em primeiro lugar, notamos que a Eq. A13 torna-se

$$
\begin{aligned}
& {\left[A_{\sigma}^{+}(x), A_{\tau}^{-}\left(x^{\prime}\right)\right]} \\
& \quad=i \sum_{j, l=1}^{2} \bar{g}_{j l} \varepsilon_{\sigma}^{(j)} \otimes \varepsilon_{\tau}^{\prime(l)} D_{2}^{+(\bmod )}\left(\widetilde{x}-\widetilde{x}^{\prime} ; x_{3}, x_{3}^{\prime}\right),
\end{aligned}
$$

com a função acima dada por

$$
\begin{aligned}
D_{2}^{+(\text {mod })} & \left(\widetilde{x}-\widetilde{x}^{\prime} ; x_{3}, x_{3}^{\prime}\right) \\
\quad & i \int \frac{d^{3} k}{(2 \pi)^{3} k_{0}} \cos \left(k_{3}\left(x_{3}-d\right)\right) \cos \left(k_{3}\left(x_{3}^{\prime}-d\right)\right) \\
\quad & \times \exp \left[-i \widetilde{k}\left(\widetilde{x}-\widetilde{x}^{\prime}\right)\right] .
\end{aligned}
$$

Utilizando a fórmula de Poisson A26 torna-se

$$
\begin{aligned}
& D_{c s}^{\text {mod }}\left(\tilde{x}-\tilde{x}^{\prime} ; x_{3}, \tilde{x}_{3}\right) \\
& \quad=\left[D_{0}^{+}\left(x-x^{\prime}\right)+D_{0}^{+}\left(x-x_{2 d}^{\prime \prime}\right)\right],
\end{aligned}
$$

com o ponto $x_{2 d}^{\prime \prime}$ significando

$$
x_{2 d}^{\prime \prime}=\left(x_{0}^{\prime}, x_{1}^{\prime}, x_{2}^{\prime},-x_{3}^{\prime}+2 d\right) .
$$

A reescrita de $D_{2}^{+(\text {mod })}$ em A27) quando comparada àquela de (A15) revela que a diferença entre as expressões é por uma contante, precisamente $-2 d$. Sendo assim, o resultado em A24 mantém-se, observando agora que $x_{3}=d$, ou seja,

$$
\begin{aligned}
p(\tilde{x}, d) & =\lim _{\delta \rightarrow 0}\left[\left\langle T_{33}^{\mathrm{FIS}}\left(\widetilde{x}, x_{3}=d+\delta\right)\right\rangle\right. \\
& \left.-\left\langle T_{33}^{\mathrm{FIS}}\left(\widetilde{x}, x_{3}=d-\delta\right)\right\rangle\right]=0 .
\end{aligned}
$$

Portanto, os resultados obtidos para o vínculo condutor sobre um plano são os esperados pela simetria do problema, ou seja, a pressão resultante é igual a zero, seja em $x_{3}=0$ ou $x_{3}=d$.

\section{O Vínculo Permeável Simples (PS)}

O espaço encontra-se dividido em duas regiões desconexas pelo plano infinitamente permeável. É a condição de fronteira de Neumann sobre o plano, que segue da Eq. (4),

$$
\begin{array}{r}
\left.\frac{\triangle_{\perp}}{\sqrt{\triangle_{\perp} \triangle}} \partial_{0} \mathrm{fs}_{(2)}(x)\right|_{x_{3}=0}=0, \\
\frac{\triangle_{3}}{\sqrt{\triangle_{\perp} \triangle_{3}}} \partial_{2} \mathrm{f}^{\mathrm{ps}}{ }_{(1)}(x)-\left.\frac{\triangle}{\sqrt{\triangle_{\perp} \triangle}} \partial_{1} \mathrm{fs}_{(2)}(x)\right|_{x_{3}=0}=0, \\
\frac{\triangle_{3}}{\sqrt{\triangle_{\perp} \triangle_{3}}} \partial_{1} \mathrm{f}^{\mathrm{ps}}{ }_{(1)}(x)+\left.\frac{\triangle}{\sqrt{\triangle_{\perp} \triangle}} \partial_{2} \mathrm{f}^{\mathrm{ps}}{ }_{(2)}(x)\right|_{x_{3}=0}=0,
\end{array}
$$

e sendo as funções, $\mathrm{f}^{\mathrm{ps}}{ }_{(j)}(x)$ independentes uma das outras conseguimos

$$
\left.\mathrm{f}^{\mathrm{ps}}(j)(x)\right|_{x_{3}=0}=0, \quad \text { para } \quad(j=1,2) .
$$

A expressão do campo de radiação sujeito ao vínculo A30 assume, diferenciando as regiões $D$ e $E$ nas quais o espaço fica dividido através 
do subscrito $\chi$, a seguinte forma

$$
\begin{aligned}
A_{\mu}(x) & =\sum_{p=1,2} \varepsilon_{\mu}^{(p)} \int \frac{d^{3} k}{(2 \pi)^{3 / 2} \sqrt{k_{0}}} \operatorname{sen}\left(k_{3} x_{3}\right) \times \\
& \times\left[\exp (-i \widetilde{k} \widetilde{x}) a_{(p) \chi}(\mathbf{k})+\exp (i \widetilde{k} \widetilde{x}) a_{(p) \chi}^{\dagger}(\mathbf{k})\right] .
\end{aligned}
$$

Seguindo o procedimento colocado na seção anterior, calculamos o primeiro termo entre parênteses na expressão do tensor em A2. Para o vínculo em questão temos

$$
\begin{aligned}
A_{\sigma}(x) A_{\tau}\left(x^{\prime}\right) & =\bullet A_{\sigma}(x) A_{\tau}\left(x^{\prime}\right) \bullet \\
& +\left[A_{\sigma}^{+}(x), A_{\tau}^{-}\left(x^{\prime}\right)\right],
\end{aligned}
$$

onde • • na equação acima é a notação para o produto normal entre operadores com respeito ao estado de vácuo $\left|0_{p s}\right\rangle$. Calculando o comutador em A33 obtemos

$$
\begin{aligned}
& {\left[A_{\sigma}^{+}(x), A_{\tau}^{-}\left(x^{\prime}\right)\right]} \\
& \quad=i \sum_{j, l=1}^{2} \bar{g}_{j l} \varepsilon_{\sigma}^{(j)} \otimes \varepsilon_{\tau}^{\prime(l)} D_{1}^{+}\left(\widetilde{x}-\widetilde{x}^{\prime} ; x_{3}, x_{3}^{\prime}\right),
\end{aligned}
$$

onde $D_{1}^{+}\left(\widetilde{x}-\widetilde{x}^{\prime} ; x_{3}, x_{3}^{\prime}\right)$ é a função

$$
\begin{aligned}
D_{1}^{+} & \left(\widetilde{x}-\widetilde{x}^{\prime} ; x_{3}, x_{3}^{\prime}\right) \\
& =i \int \frac{d^{3} k}{(2 \pi)^{3} k_{0}} \operatorname{sen}\left(k_{3} x_{3}\right) \operatorname{sen}\left(k_{3} x_{3}^{\prime}\right) \times \\
& \times \exp \left[-i \widetilde{k}\left(\widetilde{x}-\widetilde{x}^{\prime}\right)\right],
\end{aligned}
$$

que também pode ser reescrita usando a fórmula de Poisson [10],

$$
D_{1}^{+}\left(\tilde{x}-\tilde{x}^{\prime} ; x_{3}, \tilde{x}_{3}\right)=D_{0}^{+}\left(x-x^{\prime}\right)-D_{0}^{+}\left(x-x^{\prime \prime}\right),
$$

Os próximos passos são análogos àqueles entre as equações (A15) e (A23), exceto que o sinal da contribuição devida a $D_{0}^{+}\left(x-x^{\prime \prime}\right)$ é agora negativo. Entretanto, isto não altera o resultado em A23 e conduz, portanto, à Eq. A24 também para este caso

$$
\left\langle T_{33}^{\mathrm{FIS}}(x)\right\rangle_{c s}=0 .
$$

Naturalmente, a pressão de Casimir para o caso do plano infinitamente permeável mantém o resultado encontrado em (61).

Vamos detalhar agora as modificações resultantes quando deslocamos o plano para $x_{3}=d$. Em lugar de A34 temos agora

$$
\begin{aligned}
& {\left[A_{\sigma}^{+}(x), A_{\tau}^{-}\left(x^{\prime}\right)\right]} \\
& =i \sum_{j, l=1}^{2} \bar{g}_{j l} \varepsilon_{\sigma}^{(j)} \otimes \varepsilon_{\tau}^{\prime(l)} D_{1}^{+(m o d)}\left(\widetilde{x}-\widetilde{x}^{\prime} ; x_{3}, x_{3}^{\prime}\right),
\end{aligned}
$$

onde a função $D_{1}^{+(\bmod )}\left(\widetilde{x}-\widetilde{x}^{\prime} ; x_{3}, x_{3}^{\prime}\right)$ é

$$
\begin{aligned}
& D_{1}^{+(\text {mod })}\left(\widetilde{x}-\widetilde{x}^{\prime} ; x_{3}, x_{3}^{\prime}\right) \\
& =i \int \frac{d^{3} k}{(2 \pi)^{3} k_{0}} \operatorname{sen}\left(k_{3}\left(x_{3}-d\right)\right) \operatorname{sen}\left(k_{3}\left(x_{3}^{\prime}-d\right)\right) \\
& \times \exp \left[-i \widetilde{k}\left(\widetilde{x}-\widetilde{x}^{\prime}\right)\right] .
\end{aligned}
$$

Usando a fórmula de Poisson A39 torna-se

$$
\begin{aligned}
D_{p s}^{\text {mod }} & \left(\tilde{x}-\tilde{x}^{\prime} ; x_{3}, \tilde{x}_{3}\right) \\
\quad= & {\left[D_{0}^{+}\left(x-x^{\prime}\right)-D_{0}^{+}\left(x-x_{2 d}^{\prime \prime}\right)\right] . }
\end{aligned}
$$

A pressão de Casimir, que é a pressão resultante sobre o plano $x_{3}$, naturalmente, mantém o resultado já obtido pela Eq. A29.

\section{APÊNDICE B: A FÓRMULA DE POISSON E AS FUNÇÕES PARCIAIS DE JORDAN-PAULI}

As funções que denominamos parciais de Jordan-Pauli e consideradas neste trabalho correspondem às parcelas de frequência positiva das funções de Jordan-Pauli [1],

$D_{0}\left(\tilde{x}-\tilde{x}^{\prime} ; x_{3}, \tilde{x}_{3}\right)=D_{0}^{+}\left(x-x^{\prime}\right)+D_{0}^{-}\left(x-x_{2 d}^{\prime}\right)$.

A função de Green do fóton na ausência de vínculos $D_{0 \mu \nu}\left(x-x^{\prime}\right)$ é escrita, em função de $D_{0}^{+}\left(x-x^{\prime}\right)$, como segue,

$$
\begin{aligned}
D_{0 \mu \nu}\left(x-x^{\prime}\right) & =-i g_{\mu \nu}\left[\theta\left(t-t^{\prime}\right) D_{0}^{+}\left(x-x^{\prime}\right)\right. \\
& \left.-\theta\left(t^{\prime}-t\right) D_{0}^{-}\left(x-x^{\prime}\right)\right], \quad(\mathrm{B} 2)
\end{aligned}
$$


com

$$
D_{0}^{-}\left(x-x^{\prime}\right)=-D_{0}^{+}\left(x^{\prime}-x\right)
$$

Na representação do espaço dos momentos, a função de Green em (B2) é [18],

$D_{0 \mu \nu}\left(x-x^{\prime}\right)=-\frac{g_{\mu \nu}}{(2 \pi)^{4}} \int d^{4} k \frac{\exp \left[-i k\left(x-x^{\prime}\right)\right]}{k^{2}+i \eta}$,

enquanto que no espaço de coordenadas,

$$
D_{0 \mu \nu}\left(x-x^{\prime}\right)=\frac{g_{\mu \nu}}{4 i \pi^{2}}\left[\left(x-x^{\prime}\right)^{2}-i \eta\right]^{-1} \text {. }
$$

Já a função $D_{0}^{+}\left(x-x^{\prime}\right)$ no espaço dos momentos tem como expressão

$$
D_{0}^{+}\left(x-x^{\prime}\right)=\frac{1}{(2 \pi)^{3}} \int \frac{d^{3} \mathbf{k}}{2 k_{0}} \exp \left[-i k\left(x-x^{\prime}\right)\right] .
$$

Nosso objetivo é encontrar a escrita das funções (45), (69) e (79) em termos da função (B6). Iniciamos com a função $D_{c d}$ em 45 ,

$$
\begin{aligned}
& D_{c d}\left(\widetilde{x}-\widetilde{x}^{\prime} ; x_{3}, x_{3}^{\prime}\right) \\
& =i \sum_{n=1}^{\infty} \int \frac{d^{2} k_{\perp}}{(2 \pi)^{2} k_{0} d} \cos \left[\frac{n \pi x_{3}}{d}\right] \cos \left[\frac{n \pi x_{3}^{\prime}}{d}\right] \times \\
& \times \exp \left(-i \widetilde{k}\left(\widetilde{x}-\widetilde{x}^{\prime}\right)\right) .
\end{aligned}
$$

Sabemos que $\cos (x)$ é uma função par $(x \in$ $\mathbb{R})$, com isso podemos reescrever o produto de cossenos como segue

$$
\begin{aligned}
& D_{c d}\left(\widetilde{x}-\widetilde{x}^{\prime} ; x_{3}, x_{3}^{\prime}\right) \\
& \quad=i \sum_{n=-\infty}^{\infty} \int \frac{d^{3} \mathbf{k}}{2(2 \pi)^{2} k_{0} d} \exp \left(-i \widetilde{k}\left(\widetilde{x}-\widetilde{x}^{\prime}\right)\right) \times \\
& \times \delta\left(k_{3}-\left(\frac{n \pi}{d}\right)\right) \cos \left(k_{3} x_{3}\right) \cos \left(k_{3} x_{3}^{\prime}\right) \cdot(\mathrm{B} 8)
\end{aligned}
$$

A função delta na Eq. (B8) torna-se, usando a fórmula de soma de Poisson,

$$
\sum_{n=-\infty}^{\infty} f(2 n \pi)=\frac{1}{(2 \pi)} \sum_{l=-\infty}^{\infty} \int_{-\infty}^{\infty} d k f(k) \exp (-i l k)
$$

e assim, o somatório das funções delta na Eq. (B8) resulta,

$\sum_{n=-\infty}^{\infty} \delta\left(k_{3}-\left(\frac{n \pi}{d}\right)\right)=\frac{d}{\pi} \sum_{l=-\infty}^{\infty} \exp \left(-2 i k_{3} l d\right)$.

O produto de cossenos, lembrando da fórmula de Euler, assume a seguinte forma

$$
\cos \left(k_{3} x_{3}\right) \cos \left(k_{3} x_{3}^{\prime}\right)=\frac{1}{4}[A+B-C-D],
$$

com os termos $A, B, C$ e $D$ dados por

$$
\begin{aligned}
& A=\exp \left(i k_{3}\left(x_{3}-x_{3}^{\prime}\right)\right), \\
& B=\exp \left(-i k_{3}\left(x_{3}-x_{3}^{\prime}\right)\right), \\
& C=\exp \left(i k_{3}\left(x_{3}+x_{3}^{\prime}\right)+i \pi\right), \\
& D=\exp \left(-i k_{3}\left(x_{3}+x_{3}^{\prime}\right)-i \pi\right) .
\end{aligned}
$$

Substituindo (B10) e B11 na Eq. B8,

$$
\begin{aligned}
& D_{c d}\left(\widetilde{x}-\widetilde{x}^{\prime} ; x_{3}, x_{3}^{\prime}\right) \\
& \quad=i \frac{1}{(2 \pi)^{3}} \sum_{l=-\infty}^{\infty} \int \frac{d^{3} \mathbf{k}}{4 k_{0}} \exp \left(-i \widetilde{k}\left(\widetilde{x}-\widetilde{x}^{\prime}\right)\right) \\
& \times \exp \left(-2 i k_{3} l d\right)[A+B-C-D] .
\end{aligned}
$$

Efetuamos agora a troca do sinal do índice $l \rightarrow$ $-l$ nos termos $A$ e $C$ e a mudança do sinal de $k_{3} \rightarrow-k_{3}$ nos termos $B$ e $D$,

$$
\begin{aligned}
& D_{c d}\left(\widetilde{x}-\widetilde{x}^{\prime} ; x_{3}, x_{3}^{\prime}\right) \\
& =i \frac{1}{(2 \pi)^{3}} \sum_{l=-\infty}^{\infty} \int \frac{d^{3} \mathbf{k}}{2 k_{0}} \exp \left(-i \widetilde{k}\left(\widetilde{x}-\widetilde{x}^{\prime}\right)\right) \times \\
& \times[\bar{A}-\bar{B}],
\end{aligned}
$$

com

$$
\begin{aligned}
& \bar{A}=\exp \left(i k_{3}\left(x_{3}-x_{3}^{\prime}+2 l d\right)\right), \\
& \bar{B}=\exp \left(i k_{3}\left(x_{3}+x_{3}^{\prime}+2 l d\right)\right),
\end{aligned}
$$

ou seja, observando a Eq. (B6) resulta

$$
\begin{aligned}
& D_{c d}\left(\widetilde{x}-\widetilde{x}^{\prime} ; x_{3}, x_{3}^{\prime}\right) \\
& \quad=\sum_{l=-\infty}^{\infty}\left[D_{0}^{+}\left(x-x_{d}^{\prime}\right)+D_{0}^{+}\left(x-x_{d}^{\prime \prime}\right)\right],
\end{aligned}
$$


com os pontos $x^{\prime}$ e $x^{\prime \prime}$

$$
\begin{aligned}
& x_{d}^{\prime}=\left(x_{0}^{\prime}, x_{1}^{\prime}, x_{2}^{\prime}, x_{3}^{\prime}-2 l d\right), \\
& x_{d}^{\prime \prime}=\left(x_{0}^{\prime}, x_{1}^{\prime}, x_{2}^{\prime},-x_{3}^{\prime}-2 l d\right) .
\end{aligned}
$$

Passamos agora à função $D_{p d}$ em $(69)$,

$$
\begin{aligned}
& D_{p d}\left(\widetilde{x}-\widetilde{x}^{\prime} ; x_{3}, x_{3}^{\prime}\right) \\
& =i \sum_{n=0}^{\infty} \int \frac{d^{2} k_{\perp}}{(2 \pi)^{2} k_{0} d} \operatorname{sen}\left[\frac{n \pi x_{3}}{d}\right] \operatorname{sen}\left[\frac{n \pi x_{3}^{\prime}}{d}\right] \times \\
& \quad \times \exp \left(-i \widetilde{k}\left(\widetilde{x}-\widetilde{x}^{\prime}\right)\right) .
\end{aligned}
$$

O produto de senos é uma função de paridade par, assim B21 torna-se,

$$
\begin{aligned}
& D_{p d}\left(\widetilde{x}-\widetilde{x}^{\prime} ; x_{3}, x_{3}^{\prime}\right) \\
& =i \sum_{n=-\infty}^{\infty} \int \frac{d^{3} \mathbf{k}}{2(2 \pi)^{2} k_{0} d} \exp \left(-i \widetilde{k}\left(\widetilde{x}-\widetilde{x}^{\prime}\right)\right) \\
& \times \delta\left(k_{3}-\left(\frac{n \pi}{d}\right)\right) \sin \left(k_{3} x_{3}\right) \sin \left(k_{3} x_{3}^{\prime}\right) .
\end{aligned}
$$

Por sua vez, o produto de senos em termos de exponenciais assume a forma

$$
\sin \left(k_{3} x_{3}\right) \sin \left(k_{3} x_{3}^{\prime}\right)=\frac{1}{4}[E+F-G-H],
$$

com $E, F, G$ e $H$ dados por,

$$
\begin{aligned}
E & =\exp \left(i k_{3}\left(x_{3}-x_{3}^{\prime}\right)\right), \\
F & =\exp \left(-i k_{3}\left(x_{3}-x_{3}^{\prime}\right)\right), \\
G & =\exp \left(i k_{3}\left(x_{3}+x_{3}^{\prime}\right)\right), \\
H & =\exp \left(-i k_{3}\left(x_{3}+x_{3}^{\prime}\right)\right) .
\end{aligned}
$$

Substituindo (B10) e (B23) na Eq. B8),

$$
\begin{aligned}
& D_{p d}\left(\widetilde{x}-\widetilde{x}^{\prime} ; x_{3}, x_{3}^{\prime}\right) \\
& \quad=\frac{1}{(2 \pi)^{3}} \sum_{l=-\infty}^{\infty} \int \frac{d^{3} \mathbf{k}}{4 k_{0}} \exp \left(-i \widetilde{k}\left(\widetilde{x}-\widetilde{x}^{\prime}\right)\right) \\
& \quad \times \exp \left(-2 i k_{3} l d\right)[E+F-G-H] . \quad
\end{aligned}
$$

Em analogia à Eq. (B17) temos agora,

$$
\begin{aligned}
& D_{p d}\left(\widetilde{x}-\widetilde{x}^{\prime} ; x_{3}, x_{3}^{\prime}\right) \\
& =\frac{1}{(2 \pi)^{3}} \sum_{l=-\infty}^{\infty} \int \frac{d^{3} \mathbf{k}}{2 k_{0}} \exp \left(-i \widetilde{k}\left(\widetilde{x}-\widetilde{x}^{\prime}\right)\right) \times \\
& \times[\bar{E}-\bar{F}],
\end{aligned}
$$

onde os termos $\bar{E}$ e $\bar{F}$ dados por

$$
\begin{aligned}
\bar{E} & =\exp \left(i k_{3}\left(x_{3}-x_{3}^{\prime}+2 l d\right)\right), \\
\bar{F} & =\exp \left(i k_{3}\left(x_{3}+x_{3}^{\prime}+2 l d\right)\right) .
\end{aligned}
$$

Retomando a definição (B7), a Eq. B29 torna-se,

$$
\begin{aligned}
& D_{p d}\left(\widetilde{x}-\widetilde{x}^{\prime} ; x_{3}, x_{3}^{\prime}\right) \\
& \quad=\sum_{l=-\infty}^{\infty}\left[D_{0}^{+}\left(x-x_{d}^{\prime}\right)-D_{0}^{+}\left(x-x_{d}^{\prime \prime}\right)\right],
\end{aligned}
$$

Por fim, determinamos a representação em termos das $D_{0}^{+}$para a função 79

$$
\begin{aligned}
& D_{m t}\left(\widetilde{x}-\widetilde{x}^{\prime} ; x_{3}, x_{3}^{\prime}\right) \\
& =\sum_{n=0}^{\infty} \int \frac{d^{2} \mathbf{k}_{\perp}}{(2 \pi)^{2} k_{0} d} \cos \left[\left(n+\frac{1}{2}\right) \frac{\pi x_{3}}{d}\right] \\
& \quad \times \cos \left[\left(n+\frac{1}{2}\right) \frac{\pi x_{3}^{\prime}}{d}\right] \exp \left(-i \widetilde{k}\left(\widetilde{x}-\widetilde{x}^{\prime}\right)\right) .
\end{aligned}
$$

Seguindo em analogia ao que fizemos anteriormente, podemos reescrever B31 usando uma função delta apropriada,

$$
\begin{aligned}
& D_{m t}\left(\widetilde{x}-\widetilde{x}^{\prime} ; x_{3}, x_{3}^{\prime}\right) \\
& =\sum_{n=-\infty}^{\infty} \int \frac{d^{3} \mathbf{k}}{2(2 \pi)^{2} k_{0} d} \exp \left(-i \widetilde{k}\left(\widetilde{x}-\widetilde{x}^{\prime}\right)\right) \\
& \quad \times \delta\left(k_{3}-\left(n+\frac{1}{2}\right) \frac{\pi}{d}\right) \cos \left(k_{3} x_{3}\right) \cos \left(k_{3} x_{3}^{\prime}\right) .
\end{aligned}
$$

O produto de cossenos em (B32) desenvolve-se de acordo com (B11) enquanto que a reescrita da função delta na expressão acima segue a Eq. (B9), ou seja,

$$
\begin{aligned}
& \sum_{n=-\infty}^{\infty} \delta\left(k_{3}-\left(\frac{\left(n+\frac{1}{2}\right) \pi}{d}\right)\right) \\
& =\frac{d}{\pi} \sum_{l=-\infty}^{\infty} \exp \left[-i\left(2 l d k_{3}-\pi l\right)\right]
\end{aligned}
$$

O procedimento a seguir é análogo àquele estabelecido na obtenção das equações (B16) e (B17). Com o conjunto de alterações lá especificado, a representação da função $D_{m 1}^{+}(x)$ em 
termos da função $D_{0}^{+}$assume a forma,

$$
\begin{aligned}
D_{m t} & \left(\widetilde{x}-\widetilde{x}^{\prime} ; x_{3}, x_{3}^{\prime}\right) \\
& =\sum_{l=-\infty}^{\infty}(-1)^{l}\left[D_{0}^{+}\left(x-x^{\prime}\right)+D_{0}^{+}\left(x-x^{\prime \prime}\right)\right],
\end{aligned}
$$

com os pontos $x^{\prime}$ e $x^{\prime \prime}$ sendo aqueles definidos por $(\mathrm{B} 12)$ e $\mathrm{B} 13)$.

Completamos assim o estudo de representação das funções $D_{c d}\left(x-x^{\prime \prime}\right), D_{p d}\left(x-x^{\prime \prime}\right)$, e $D_{m t}\left(x-x^{\prime \prime}\right)$ em termos da função parcial de Jordan-Pauli $D_{0}^{+}\left(x-x^{\prime}\right)$.
[1] G. Scharf, Finite Quantum Electrodynamics - The Causal Approach. New York: Springer (1989).

[2] K.O. Friedrichs, Mathematical Aspects of the Quantum Theory of Fields. New York: Interscience (1953).

[3] H.B.G. Casimir. Proc. K. Akad. Wet. B 51, 793 (1948).

[4] G. Scharf, W.F. Wreszinski. Found. Phys. Lett. 5 , 479 (1992).

[5] G. Scharf, W.F. Wreszinski. Nuovo Cimento 107A, 2879 (1994).

[6] W.F. Wreszinski, C.D. Jäkel. Annals of Physics 323, 251 (2008).

[7] N.A. Kawakami, M.C. Nemes, W.F. Wreszinski. J. Math. Phys. 48, 102302 (2007).

[8] W.F. Wreszinski. Braz. J. Phys. 34, 1343 (2004).

[9] L.A. Manzoni, W.F. Wreszinski. Eur. Phys. J. C25, 315 (2002).

[10] F.A. Farias, Eletrodinâmica Quântica em Cavidades: o Efeito Casimir e as Correções Radiativas na Massa e no Momento Magnético Anômalo do Elétron. Dissertação (Mestrado) Instituto de Física da UFBA, Salvador (1996).

[11] V.M. Mostepanenko. Sov. Phys. - JEPT (USA) 31, 965 (1988).

[12] V.M. Mostepanenko, The Casimir Effect and its Applications. Oxford: Clarendon Press (1997).

[13] G. Plunien, B. Müller, W. Greiner. Phys. Rep. 134, 87 (1986).

[14] N.D. Birrel, P.C.W. Davies, Quantum Field Theory in Curved Spaces. Cambridge: Cambridge University Press (1982).

[15] S.A. Fulling, Aspects of Quantum Field Theory in Curved Space-Time. Cambridge: Cambridge University Press (1989).

[16] M. Bordag, U. Mohideen, V.M. Mostepanenko. Phys. Rep. 353, 1 (2001).
[17] J.D. Jackson, Classical Electrodynamics. New York: John Wiley \& Sons (1990).

[18] C. Itzykson, J.B. Zuber, Quantum Field Theory. New York: McGraw-Hill (1980).

[19] D. Robaschik, E. Wieczorek. Ann. Phys. (N.Y.) 236, 43 (1994).

[20] M. Bordag, D. Robaschik, E. Wieczorek. Ann. Phys. (N.Y.) 174, 401 (1987).

[21] M.J. Sparnaay, Measurements of Attractive Forces between Flat Plates. Physica 24, 751 (1958).

[22] S.K. Lamoreaux, Demonstration of the Casimir Force in the 0.6 to $6 \mu \mathrm{m}$ Range. Phys. Lett. 78, 5 (1997).

[23] U. Mohideen, A. Roy, Precision Measurement of the Casimir Force from 0.1 to $0.9 \mu \mathrm{m}$. Phys. Rev. Lett. 81, 4549 (1998).

[24] A. Roy, U. Mohideen, Demonstration of the Nontrivial Boundary Dependence of the Casimir Force. Phys. Rev. Lett. 82, 4380 (1999).

[25] I.S. Gradshteyn, I.M. Ryzhik, Table of Integrals, Series and Products. New York: Academic Press (1965).

[26] F. Strocchi, Elements of Quantum Mechanics of Infinity Systems. Singapore: World Scientific (1985).

[27] B.V. Deriagin, I.I. Abrikosova. Sov. Phys. Jept 3, 819 (1957).

[28] M.J. Spaarnay. Physica 24, 751 (1958).

[29] E.S. Sabisky, C.H. Anderson. Phys. Rev. A 7, 790 (1973).

[30] M. Bordag, V.M. Mostepanenko, I.Yu. Sobolev. Surface Science 328, 129 (1995).

[31] G.L. Klimchitskaya, Yu.V. Pavlov. Int. Jour. Mod. Phys. A11, 3723 (1996).

[32] L.S. Brown, G.J. Maclay. Phys. Rev. 184, 1272 (1969).

[33] T.H. Boyer. Ann. Phys. 56, 474 (1970). 